NBER WORKING PAPER SERIES

\title{
VACCINE ALLOCATION PRIORITIES USING DISEASE SURVEILLANCE AND ECONOMIC DATA
}

\author{
Anup Malani \\ Satej Soman \\ Sabareesh Ramachandran \\ Alice Chen \\ Darius N. Lakdawalla \\ Working Paper 29682 \\ http://www.nber.org/papers/w29682 \\ NATIONAL BUREAU OF ECONOMIC RESEARCH \\ 1050 Massachusetts Avenue \\ Cambridge, MA 02138 \\ January 2022
}

Malani acknowledges funding from the Becker Friedman Institute at the University of Chicago to purchase a subscription to the Consumer Pyramids Household Survey and the support of the Barbara J. and B. Mark Fried Fund at the University of Chicago Law School. We thank Maciej Boni for helpful comments. Correspondence: amalani@uchicago.edu. The views expressed herein are those of the authors and do not necessarily reflect the views of the National Bureau of Economic Research.

At least one co-author has disclosed additional relationships of potential relevance for this research. Further information is available online at http://www.nber.org/papers/w29682.ack

NBER working papers are circulated for discussion and comment purposes. They have not been peer-reviewed or been subject to the review by the NBER Board of Directors that accompanies official NBER publications.

(C) 2022 by Anup Malani, Satej Soman, Sabareesh Ramachandran, Alice Chen, and Darius N. Lakdawalla. All rights reserved. Short sections of text, not to exceed two paragraphs, may be quoted without explicit permission provided that full credit, including $(\subset)$ notice, is given to the source. 
Vaccine Allocation Priorities Using Disease Surveillance and Economic Data

Anup Malani, Satej Soman, Sabareesh Ramachandran, Alice Chen, and Darius N. Lakdawalla

NBER Working Paper No. 29682

January 2022

JEL No. I1,I18

\begin{abstract}
$\underline{\text { ABSTRACT }}$
Vaccination is a critical tool, along with suppression and treatment, for controlling epidemics such as SARS-CoV-2. To maximize the impact of vaccination, doses should be allocated to the highest value targets, accounting for health and potential economic benefits. We examine what allocation strategy is optimal and how to translate that strategy into actionable procurement decisions in the context of India. We compare 3 different allocation strategies (oldest first, highest contact rate first, random order) across 4 outcomes (lives saved, life-years saved, value of statistical lives saved, value of statistical life-years saved). We make 3 methodological contributions. First, we estimate the incremental health benefit of vaccination using novel, local seroprevalence data from India. Second, we estimate the value of statistical life-years using disaggregated, monthly data on consumption during the pandemic. Third, and most importantly, we estimate social demand curves for vaccines that can practically guide government procurement decisions. Our analysis yields 4 novel findings. First, the need to speed-up vaccination does not justify deviation from elderly-first prioritization. Second, much of the value of vaccination comes from improvements in consumption rather than longevity. Moreover, vaccination increases the value of a life year because it increases consumption. Third, social demand for vaccination falls over time as natural immunity from infections increases. Therefore, the slower a country vaccinates its population, the fewer doses it should procure. Fourth, there is enough variation in consumption and infection risk that it makes sense to vaccinate some areas before others. Our approach of connecting epidemiological models and data on health and consumption to economic valuation methods generalizes to other infection control strategies, such as suppression, and public health crises, such as influenza and HIV.
\end{abstract}

Anup Malani

University of Chicago Law School

1111 E. 60th Street

Chicago, IL 60637

and NBER

amalani@uchicago.edu

Satej Soman

School of Information

UC Berkeley

satej@berkeley.edu

Sabareesh Ramachandran

Department of Economics

UC San Diego

saramach@ucsd.edu
Alice Chen

Schaeffer Center for Health Policy and Economics University of Southern California

635 Downey Way

Los Angeles, CA 90089-3333

alicejc@usc.edu

Darius N. Lakdawalla

University of Southern California

635 Downey Way, VPD 414-K

Schaeffer Center for Health Policy and Economics

Los Angeles, CA 90089-7273

and NBER

dlakdawa@usc.edu 


\section{Introduction}

The SARS-CoV-2 (COVID-19) pandemic has infected over 250 million and killed at least 5 million worldwide. Nearly 2 years into the crisis, many countries, such as India, have experienced second waves with infection levels greater than the initial wave (Johns Hopkins University \& Medicine, 2021) and now face a potential third wave from the Omicron variant that is larger still. Lacking an effective medical treatment, countries initially relied on non-pharmaceutical interventions to contain the epidemic (Brauner et al., 2021). This involves a trade-off between health and economic activity (Norheim et al., 2021). After 1 year, vaccines had been approved that could both contain the epidemic and allow socioeconomic activity to resume. However, there continue to be shortages in vaccine availability (Duke Global Health Innovation Center, Duke Global Health Innovation Center). Although it is difficult to budge existing vaccination campaigns, a critical question is what allocation would have done a better job maximizing the health and economic benefits from vaccination.

Prior analyses of optimal vaccine allocation typically begin with a model of disease, then simulate or forecast the effect of various vaccine allocation plans, and finally compare plans based on certain metrics. Disease is commonly forecast from a compartmental model (Bubar et al., 2021. Buckner et al., 2021; Jentsch et al., 2021; Agarwal et al., 2021; Kirwin et al., 2020), but some papers also employ a constant hazard rate (Babus et al., 2020; Chapman et al., 2021; Goldstein et al., 2021) estimated from local data or an agent-based model (Chen et al., 2021). Given the higher infection fatality rate (IFR) for the elderly, all papers consider a plan that prioritizes the elderly. Many papers also consider plans that prioritize other groups with high IFR (Babus et al., 2020; Chapman et al. 2021; Kohli et al., 2021) or contact rate (Buckner et al., 2021; Jentsch et al., 2021; Chapman et al. 2021; Chen et al., 2021; Mulberry et al., 2021). A small number optimize plans (Buckner et al., 2021; Matrajt et al., 2020; Rodríguez et al., 2020) rather than compare pre-specified plans. Others papers compare specified plans to a benchmark such as uniform allocation (Bubar et al. 2021; Jentsch et al., 2021; Chapman et al., 2021) or no vaccination (Kirwin et al., 2020). Nearly all papers compare plans on deaths averted and years of life saved. Some also look at the impact on infections (Bubar et al., 2021; Buckner et al., 2021; Chapman et al., 2021; Chen et al., 2021) and hospitalizations (Chen et al., 2021; Kirwin et al., 2020). A few papers examine cost-effectiveness (Kohli et al., 2021) or monetary benefit (Kirwin et al., 2020, Mulberry et al., 2021).

Here we build on prior evaluations of vaccine allocation in three important respects. First, 
we use a range of novel epidemiological data from a low-to-middle income country, India, where a second wave of SARS-CoV-2 has already claimed over 250,000 lives and vaccines are in short supply covid19india.org, 2021), to parameterize a local, compartmental model of infection. Specifically, we use the results of a new population-level serological survey (Selvavinayagam et al., 2021) that sampled 26,640 persons across every district in the state of Tamil Nadu (population 67 million). The state had a seroprevalence of $67 \%$ in July 2021 after India's second wave. Seroprevalence varied substantially $(64.1-74.8 \%)$ by urbanization rates, a factor used to extrapolate the results to districts in other states. Using local trends in confirmed cases, we project out recovered populations to June 15, 2022. These projections are combined with confirmed deaths to calculate local infection fatality rates. In addition, we use local contact-tracing data (Laxminarayan et al., 2020) estimate an age-mixing matrix.

Second, we generate a robust economic valuation of vaccination plans based on willingness to pay for longevity (Garber and Phelps, 1997; Murphy and Topel, 2006). We employ a geographicallyrepresentative, panel data set on consumption among 174,000 Indian households (1.2 million persons) across India through July 2021 to estimate local consumption, and thus willingness-to-pay. We use a prediction model that maps infection onto consumption to estimate how willingness-to-pay changes with different vaccination plans.

Third, we develop and calculate a model for social demand for vaccination that can guide governments' vaccine procurement decisions. Social demand is equated with the sum of private willingness-to-pay to different vaccination polices and rates. Importantly, the demand curve shifts down as more people are vaccinated and infected because others' vaccination and natural immunity reduces the value of own vaccination. Moreover, a faster vaccination plan has greater value, not merely because of time discounting, but also because faster vaccination slows the rate at which demand falls as it leaves less time for natural immunity to spread.

This paper presents tools that can provide actionable policy advice. Our estimates can help government select optimal vaccination plans on a range of metrics. Importantly, our metrics consider economic factors that influence politicians, even though they may not be what the public health community recommends. Most importantly, our demand-curve estimates recommends how many doses would be cost effective for governments to procure at different level of vaccine efficacy and price.

While our analysis focuses on the initial round of vaccination in India, it has continued relevance. India is on pace to vaccinate roughly half of its population per year, meaning prioritization is 
relevant for the half of the population that has not yet received 2 doses. Moreover, because immunity wanes, the tools we present for economic valuation and procurement are useful for prioritizing the boosters required to provide continued protection from COVID-19.

We conduct our main analysis by aggregating district-specific models at the state level and national levels in India, which ranks third in total number of cases as of this writing (Worldometers, 2021). Our framework can be applied outside India with requisite data.

\section{Data and Methods}

We use epidemiological and economic models, combined with new data on COVID-19 prevalence and economic activity, to evaluate different vaccine priority strategies for non-frontline workers in India. Here we provide a brief sketch of our methods. Additional details are in the Supplemental Materials.

Epidemic forecasting. We employ a stochastic compartmental SIRD model ((1), (2), (3), (4)) to forecast the epidemic without vaccination for 7 age categories in each district in India.

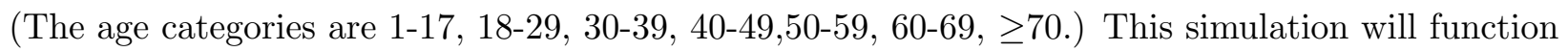
as the control group against which we evaluate all vaccination policies. In this base model, the epidemic unfolds separately in each district with no migration across districts of a state. The model is simulated in each location for the overall population of a district. At each simulation time step, daily cases, deaths and recoveries are assigned to different age categories depending on the agedistribution observed in data, and mortality is set to an infection-weighted average of age-specific mortality rates.

Model parameters are estimated from local data in India. Current reproductive rates in each district are estimated using daily, district-level data on new cases and deaths (covid19india.org, 2021) and Bayesian methods from Bettencourt \& Soman (2021) (Bettencourt and Soman, 2020). (Using Cori et al. (2013) (Cori et al., 2013) does not meaningfully change our estimates of the current reproductive rate $R_{t}$.) Infection fatality rates are borrowed from the metanalysis in O'Driscoll (2020) (O'Driscoll et al., 2020). Age-mixing is parameterized with local contract tracing data (Laxminarayan et al., 2020). For Tamil Nadu, seroprevalence as of 20 November 2020 is estimated from a new serological survey of a population-representative sample with $N=26,140$ persons from 19 October - 30 November 2020 (Malani et al. 2021). For other states, seroprevalence on that date is estimated by re-weighting the Tamil Nadu estimates to reflect demographics (e.g., age, sex) 
and urbanization in local populations (Government of India, 2011). Current recovered population is extrapolated from seroprevalance by, first, vertically shifting up the current infection curve to match the level of of infection on 30 November 2020 implied by seroprevalence surveys and, second, by projecting out new infections to 15 April 2021 based on this "lifted" new cases curve. Recovery rates are assumed to be 0.1 (Byrne et al., 2020). Allocation of cases, deaths and immunity to age bins is based on age bin shares observed in seroprevalence and official deaths data in Tamil Nadu.

To model vaccination, we define each prior compartment to be unvaccinated and introduce 6 new compartments: vaccinated with an effective dose, vaccinated with an ineffective dose, infected and vaccinated, vaccinated and then recovered, recovered and then vaccinated, and vaccinated and then dead (Figure A1). We assume that the government does not know the infection states of individuals before they are vaccinated, so all individuals in a location are vaccinated at the same rate; no individuals are vaccinated wit a full regimen twice. We assume vaccination has $70 \%$ efficacy, based on estimates of efficacy of the Astra Zeneca vaccine, one of two approved in India (Voysey et al., 2021). The vaccination rate is given by vaccination strategy.

Vaccination strategies. We examine 12 vaccination strategies. The vaccination strategies vary on (a) who is prioritized and (b) the daily vaccination rate. The first schedule prioritizes higher IFRs within districts and thus higher ages first: 70+, 60-69, 50-59, etc. (Cai et al., 2021). The second prioritizes by contact rates within districts, thus working age populations first: 18-29, 30-39, 40-49, 50-59, 0-17, 60-69, 70+. Contact rates are obtained from a large $(\mathrm{N} \approx 500,000)$ database of contact tracing efforts in Andhra Pradesh and Telangana states (Laxminarayan et al., 2020). The third distributes vaccines randomly. We examine each schedule at 4 rates: a daily rate of vaccination that would result in $25 \%, 50 \%, 100 \%$ and $200 \%$ of the population fully vaccinated every 12 months. (200\% vaccination rate is equal to $100 \%$ vaccinated in 6 months.) Being vaccinated implies getting all recommended doses. Vaccination is assumed to continue until everyone in the population is vaccinated, whichever comes first. Although the primary vaccines used in India are only partially effective (70 - 80\%) at stopping serious symptomatic infection (Voysey et al., 2021), they are nearly $100 \%$ effective at preventing death, the only outcome our valuations weight. Therefore, our model assumes a vaccine that is perfectly effective at preventing mortality. Importantly, by comparing random allocation at a high vaccination rate to, e.g., age-prioritization at a lower rate, our approach also allows us to value speed versus prioritized allocation.

Consumption forecasting. We use data from the Centre for Monitoring Indian Economy's Consumer Pyramids Household Survey, a population-representative panel survey in India covering 
approximately 174,000 households (or 1.2 million people) to forecast age bin by district-level, daily consumption. To do so we obtain monthly household-level consumption from January 2018 January 2020 and October 2020 - March 2021 from these data. We omit data during the national lockdown and the months following it, when lockdown was unwound, because we assume there will be no national lockdown in from 15 June 2021 - 15 June 2022, the period of our simulation. For our prediction model, we regress district-level monthly consumption against the level of infection, the level of deaths, month-of-year fixed effects and district fixed effects. The regression is structured such that, when COVID-19 cases vanish, consumption will return to average 2019 levels before growing again. We forecast household consumption using this regression and forecasts of local infection and death rates from our epidemiological simulations. In this forecast, aggregate levels of immunity increase everyone's income, and individual vaccination increases individual income. We assign household consumption to age bins based on the OECD's modified formula (Hagenaars et al., 1994). Our forecasts for the epidemic and consumption start on 15 June 2021.

Simulation. We run 1000 simulations of each policy (including no vaccination) in each state. Aggregate results for India are the sum of state-level simulations. The simulations assume new case counts $\Delta T_{t}$ obey a Poisson process with a time-dependent mean parameter $\lambda_{t}=\Delta T_{t-1}$. $\exp \left(\gamma \cdot\left(R_{t}-1\right)\right)$, where $\gamma$ is the recovery rate. For each simulation we project populations in each compartment for 5 years. We project consumption in each simulation based on the mean projection of our consumption forecast given forecasted infections and deaths.

Evaluating strategies. All vaccination policies are evaluated relative to a counterfactual of no vaccination and, implicitly, unchanged suppression policy and voluntary distancing. The evaluations are based on the mean outcome across all simulations of a given policy.

We initially compare strategies by summing the number of lives and number of life-years lost under each strategy. We monetize these health benefits by multiplying deaths and years of life lost by the value of a statistical life (VSL) or life-year (VSLY). VSL and VSLY are calculated by calculating the net present value of future consumption accounting for survival risk, growth, and the lifetime profile of consumption and multiplying by a constant equal to 3 (Lakdawalla and Phelps, 2020).

Finally, we calculate the "total economic value" (TEV) of each policy, defined as the aggregate willingness to pay (WTP) for increases in longevity and consumption equal to that achieved under that policy. To do so, we employ an economic model of lifetime utility (Murphy and Topel, 2006). WTP is equal to the ratio of the marginal utilities of longevity and consumption (Rosen, 1981). 
Under common assumptions, WTP is proportional to net present value of expected consumption (Lakdawalla and Phelps, 2020; Becker et al., 2005; Garber and Phelps, 1997), where expectations reflect survival probabilities obtained from our epidemic forecasting. The critical difference between VSLY and TEV is that the latter allows willingness to pay for longevity to rise consumption rises.

Decomposing total economic value. We decompose TEV into value due to improvements in survival probability holding consumption constant at post vaccination levels versus improvements in consumption holding survival at pre-vaccination levels. We also decompose full economic value into private and social value from vaccination in two steps. First, we equate per capita private value to how much an average individual in a given age and location bin is willing to pay to be vaccinated given a random allocation policy with a 50\% vaccination rate. Second, we subtract private value from TEV for the average person in that bin to obtain the per capita social value of vaccination in that bin.

Developing priority lists. We develop priority lists for different age groups in each district using the full economic value of vaccination. We develop these lists for the random assignment strategy so that we are able to also determine how much improvement different prioritization strategies offer over no prioritization at all.

Dynamic social demand. For each timestamp (a day) under a given vaccination policy, we set the value obtained by the number of people vaccinated that day equal to the average full economic value obtained those vaccinated that day. The dynamic social demand for a policy simulation sorts individuals in the order in which they are vaccinated and is equal to a step function that traces out the value obtained by those people. The dynamic social demand for a policy is the value average of these simulation-specific demands.

\section{Results}

India was hit in March 2021 with a second wave of SARS-CoV-2 that generated peak confirmed active infections far greater than the country's first wave in 2020 (Figure 1a). Vaccination began in January 2021. By mid-December 2021 roughly $60 \%$ of the population had been vaccinated with at least 1 dose, and 40\% were fully vaccinated (Hannah Ritchie and Roser, 2020) (Figure 1b). Based on estimated reproductive rates through July, our compartmental model and our consumption prediction model, we forecast that economic activity will not resume until mid-year 2022. Our analysis does not include a possible third wave due to the Omicron variant because we do not have 
data on that variant. Figures in the main text highlight India and one state, Tamil Nadu, because we have recent data on sero-positivity from that state. We also provide analysis for four other geographically-dispersed states beside Tamil Nadu to show local applicability.

Health benefits. These trends imply that the health benefits of vaccination, measured in deaths or years of of life lost (YLL) (Figures 2a and 2b), across India could be substantial. Relative to no vaccination, a vaccination rate of $50 \%$ the population per year using mortality-prioritization would save nearly 1 million lives and 10 million life-years across India.

Mortality-rate prioritization saves the most lives and life-years; its value rises sharply with the vaccination rate because it captures the most vulnerable. Random assignment saves far fewer lives over no vaccination; its value increases moderately with the vaccination rate. Contact-rate prioritization actually does slightly worse than random assignment because it initially avoids the elderly and thus most vulnerable.

Monetized health benefits. Prioritization based on monetized benefits are similar to those based on health benefits. Monetized benefits are equal to health benefits scaled by VSL or VSLY (Figures A3a and A3b), which in turn are estimated from current local consumption. Because mortality-rate prioritization vaccinates older age populations before working age ones and working age populations have higher consumption, the discounted consumption gain (and thus VSLY) for mortality-rate prioritization is better than random allocation, but not by as much when examining deaths or years YLL. Contact-rate prioritization generates the lowest gains in VSL and VSLY because it prioritizes 18-29 year-olds first; they are a large fraction of the population, have both lower infection fatality rate (IFR) and have lower consumption than even the elderly.

Economic valuation. The TEV of vaccination tracks value under VSLY, but allows for the value of life years to increase as consumption increases when infections decline (Figure 1d). TEV gives greater weight to places and ages with larger declines in consumption during the pandemic; the larger declines are increasing functions of both the pre-pandemic level of consumption and the decline in consumption during the pandemic (Figure 2d). While the rank ordering of vaccination strategies by TEV (Figure 2c) is similar to valuation based on VSLY, the incremental benefit of mortality-rate prioritization over other schemes is smaller because the latter prioritize vaccinating working-age populations that had larger pre-pandemic income and experience bigger gains in income when they are vaccinated. This additional income translates into an increase in the value of life under the TEV. The gap between random allocation and contact-rate prioritization disappears under TEV for the same reason. 
The vast majority of the TEV from vaccination accrues from improvement in consumption with amelioration of the epidemic rather than reduction in risk of death except for the very old, who experience very high IFRs (Figure 3a, where the y-axis is in log scale). This is a product of the large drop in income during the pandemic and the low death rate from the pandemic. COVID-19 is associated with a large increase in death rate (Malani and Ramachandran, 2021), but the base was low to begin with.

Priority schedules. Our priority schedules based on TEV for vaccinating age groups and districts suggest that age-based prioritization should prioritize those ages 60-69 higher than those 70 and above (Figure 2d). Geographic variation in the benefits of vaccination (Figure 3c) suggests that greater value from prioritizing lower ages in some regions before older persons in other regions (Figure 2d). Examples of priority lists are presented in the Appendix (Tables A3 - A7).

Social demand curves. At a price of $\$ 12.21 /$ dose, as has been estimated in India inclusive of distribution costs and wastage (Andersen et al., 2021), dynamic demand with value-maximizing mortality-rate prioritization suggests it is cost effective to vaccinate only $50 \%$ of the the population of India if the vaccination rate is $50 \%$ per year (Figure $3 \mathrm{~b}$ ). The optimal number of doses to purchase increases as the rate of vaccination rises.

\section{Discussion}

The second wave in India - and the current third wave - highlight the large risk from COVID-19. Given the country's population, a large number of lives can be spared with vaccination. Shortages of supply, however, suggest that allocation matters. Mortality-rate based prioritization may save a million more lives and 10 million life years.

The differences between allocation plans narrow when we translate lives into monetary values because value of life depends on income and working age populations earn more. The plans differ in value by only USD 10-20 billion when we account for increases in the value of life as consumption rises. A proportional change in consumption has bigger effects on working age population, which has a larger share of household consumption.

Our social demand curves show that it may be cost-effective to vaccinate - and thus to procure doses for - only a subset of the population if the rate of vaccination is low because it takes a long time to vaccinate the whole population. Vaccination campaigns are in a race against the epidemic. The slower the campaign, the fewer people campaign can vaccinate before the epidemic finishes, 
and the lower the campaign's value. The social value of vaccination and the optimum number of doses to purchase rise with the rate of vaccination. The incremental TEV from vaccinating at a faster pace provides a benchmark for the cost-effective level of investment in policies that will increase vaccination speed.

If the cost of speeding up vaccination is the inability to prioritize, however, it may be prudent in India to choose a slower but mortality-rate prioritized vaccination plan. Vaccinating just $25 \%$ of the population in a year using mortality-rate prioritization saves more lives and life-years than vaccinating even $100 \%$ of the population in 6 months using random allocation. Protecting a small number of the elderly eliminates much of the remaining mortality risk from COVID-19 in that country.

Our analysis has a number of limitations. First, our prioritization lists are static rather than optimal. We list subpopulations that deliver higher social value from vaccination assuming random assignment. A better, but more difficult-to-implement, approach would be to solve the dynamic optimization problem so that, on the optimal path, the marginal benefit to vaccinating any other subgroup is non-positive.

Second, our analysis has a fairly constrained view of the human behavioral response to vaccination. Our epidemiological model assumes that the latest estimated reproductive rate will persist into the future; however, it is possible that vaccination will increase contact rates and thus slow the resolution of the epidemic. Our consumption forecasting model implicitly accounts for both behavioral and governmental response because it is trained on 2020 data that includes that response; it thereby implicitly assumes, however, that future response to vaccination will only be mediated by daily infection and deaths (rather than also vaccination rates) and that the behavioral response to infections and deaths is constant over time.

Third, our analysis only groups individuals by age and location. Actual allocation plans also include occupations, such as healthcare works or governmental leaders, and those with co-morbidities. Our limited data do not permit evaluation of those groups. However, it may be difficult logistically to implement more fine-tuned allocation models.

Fourth, our approach does not consider the role that logistics may play in allocation. It is possible that geographic and age-based ordering is difficult to implement. We attempt to address this indirectly by comparing a higher rate of vaccination at random with prioritized (e.g., age-based) vaccination. However, speed is not the only cost of fine-tuning; there may be greater wastage or errors. 
Fifth, our approach to allocation does not directly address equity and ethics, which have important roles to play in vaccine prioritization. It is difficult to assign a quantitative value to equity, though our framework can be used to evaluate equal access across geographies with and without age-based allocation within regions. (While our data on consumption allow differentiating high and low income individuals, our data on COVID-19 cases and seroprevalence do not.) Our approach likewise can be used to evaluate ethical approaches that value lives or life-years equally (even monetarily) across age groups and locations.

\section{References}

Agarwal, N., A. Komo, C. A. Patel, P. A. Pathak, and M. U. Ünver (2021). The trade-off between prioritization and vaccination speed depends on mitigation measures. Report, National Bureau of Economic Research.

Andersen, C., K. Andrews, J. Cain, and A. Tandon (2021). South asia vaccinates against covid-19: Health system and health financing considerations. Report.

Babus, A., S. Das, and S. Lee (2020). The optimal allocation of covid-19 vaccines. medRxiv, 2020.07.22.20160143.

Becker, G. S., T. J. Philipson, and R. R. Soares (2005). The quantity and quality of life and the evolution of world inequality. American Economic Review 95(1), 277-291.

Bettencourt, L. M. and R. M. Ribeiro (2008). Real time bayesian estimation of the epidemic potential of emerging infectious diseases. PLoS One 3(5).

Bettencourt, L. M. A. and S. Soman (2020). Systems architecture for real time epidemiological prediction and control. Mansueto Institute for Urban Innovation Research Paper 26.

Brauner, J. M., S. Mindermann, M. Sharma, D. Johnston, J. Salvatier, T. Gavenčiak, A. B. Stephenson, G. Leech, G. Altman, V. Mikulik, A. J. Norman, J. T. Monrad, T. Besiroglu, H. Ge, M. A. Hartwick, Y. W. Teh, L. Chindelevitch, Y. Gal, and J. Kulveit (2021). Inferring the effectiveness of government interventions against covid-19. Science 371 (6531), eabd9338.

Bubar, K. M., K. Reinholt, S. M. Kissler, M. Lipsitch, S. Cobey, Y. H. Grad, and D. B. Larremore (2021). Model-informed covid-19 vaccine prioritization strategies by age and serostatus. Science, eabe6959. 
Buckner, J. H., G. Chowell, and M. R. Springborn (2021). Dynamic prioritization of covid-19 vaccines when social distancing is limited for essential workers. Proceedings of the National Academy of Sciences 118(16), e2025786118.

Byrne, A. W., D. McEvoy, A. B. Collins, K. Hunt, M. Casey, A. Barber, F. Butler, J. Griffin, E. A. Lane, C. McAloon, K. Brien, P. Wall, K. A. Walsh, and S. J. More (2020). Inferred duration of infectious period of sars-cov-2: rapid scoping review and analysis of available evidence for asymptomatic and symptomatic covid-19 cases. BMJ Open 10(8), e039856.

Cai, R., P. Novosad, V. Tandel, S. Asher, and A. Malani (2021). Representative estimates of covid-19 infection fatality rates from three locations in india. medRxiv, 2021.01.05.21249264.

Chapman, L. A. C., P. Shukla, I. Rodríguez-Barraquer, P. B. Shete, T. M. León, K. BibbinsDomingo, G. W. Rutherford, R. Schechter, and N. C. Lo (2021). Comparison of covid-19 vaccine prioritization strategies in the united states. medRxiv, 2021.03.04.21251264.

Chen, J., S. Hoops, A. Marathe, H. Mortveit, B. Lewis, S. Venkatramanan, A. Haddadan, P. Bhattacharya, A. Adiga, A. Vullikanti, M. L. Wilson, G. Ehrlich, M. Fenster, S. Eubank, C. Barrett, and M. Marathe (2021). Prioritizing allocation of covid-19 vaccines based on social contacts increases vaccination effectiveness. medRxiv, 2021.02.04.21251012.

Cori, A., N. M. Ferguson, C. Fraser, and S. Cauchemez (2013). A new framework and software to estimate time-varying reproduction numbers during epidemics. American journal of epidemiology $178(9), 1505-1512$.

covid19india.org (2021). Coronavirus Outbreak in India - covid19india.org. https://www. covid19india.org/. Accessed: 2021-31-01.

Duke Global Health Innovation Center. Launch \& Scale Speedometer - COVID-19. https:// launchandscalefaster.org/COVID-19. Accessed: 2021-21-02.

Garber, A. M. and C. E. Phelps (1997). Economic foundations of cost-effectiveness analysis. Journal of Health Economics 16(1), 1-31.

Goldstein, J. R., T. Cassidy, and K. W. Wachter (2021). Vaccinating the oldest against covid19 saves both the most lives and most years of life. Proceedings of the National Academy of Sciences 118(11), e2026322118. 
Government of India (2011). Census of India 2011. Census of India.

Hagenaars, A. J., K. De Vos, and M. Asghar Zaidi (1994). Poverty statistics in the late 1980s: Research based on micro-data. Publications Office of the European Union.

Hannah Ritchie, Edouard Mathieu, L. R.-G. C. A. C. G. E. O.-O. J. H. B. M. D. B. and M. Roser (2020). Coronavirus pandemic (covid-19). Our World in Data. https://ourworldindata.org/coronavirus.

Jentsch, P. C., M. Anand, and C. T. Bauch (2021). Prioritising covid-19 vaccination in changing social and epidemiological landscapes: a mathematical modelling study. The Lancet Infectious Diseases.

Johns Hopkins University \& Medicine (2021). COVID-19 Dashboard by the Center for Systems Science and Engineering (CSSE) at Johns Hopkins University (JHU). https://coronavirus. jhu.edu/map.html. Accessed: 2021-31-01.

Kirwin, E., E. Rafferty, K. Harback, J. Round, and C. McCabe (2020). A net benefit approach for the optimal allocation of a covid-19 vaccine. medRxiv, 2020.11.30.20240986.

Kohli, M., M. Maschio, D. Becker, and M. C. Weinstein (2021). The potential public health and economic value of a hypothetical covid-19 vaccine in the united states: Use of cost-effectiveness modeling to inform vaccination prioritization. Vaccine 39(7), 1157-1164.

Kumar, N., S. K. Shahul Hameed, G. R. Babu, M. M. Venkataswamy, P. Dinesh, P. Kumar Bg, D. A. John, A. Desai, and V. Ravi (2021). Descriptive epidemiology of sars-cov-2 infection in karnataka state, south india: Transmission dynamics of symptomatic vs. asymptomatic infections. EClinicalMedicine.

Lakdawalla, D. N. and C. E. Phelps (2020). Health technology assessment with risk aversion in health. Journal of Health Economics 72, 102346.

Laxminarayan, R., B. Wahl, S. R. Dudala, K. Gopal, C. Mohan B, S. Neelima, K. S. Jawahar Reddy, J. Radhakrishnan, and J. A. Lewnard (2020). Epidemiology and transmission dynamics of covid19 in two indian states. Science 370(6517), 691.

Malani, A. and S. Ramachandran (2021). Using household rosters from survey data to estimate 
all-cause mortality during covid in india. National Bureau of Economic Research Working Paper Series No. 29192.

Malani, A., S. Ramachandran, V. Tandel, R. Parasa, S. Sudharshini, V. Prakash, Y. Yoganathan, S. Raju, and T. Selvavinayagam (2021). Seroprevalence in tamil nadu in october-november 2020. MedRXiv.

Matrajt, L., J. Eaton, T. Leung, and E. R. Brown (2020). Vaccine optimization for covid-19: who to vaccinate first? medRxiv, 2020.08.14.20175257.

Mulberry, N., P. Tupper, E. Kirwin, C. McCabe, and C. Colijn (2021). Vaccine rollout strategies: The case for vaccinating essential workers early. medRxiv, 2021.02.23.21252309.

Murphy, K. M. and R. H. Topel (2006). The value of health and longevity. Journal of Political Economy $114(5)$.

Norheim, O. F., J. M. Abi-Rached, L. K. Bright, K. Bærøe, O. L. M. Ferraz, S. Gloppen, and A. Voorhoeve (2021). Difficult trade-offs in response to covid-19: the case for open and inclusive decision making. Nature Medicine 27(1), 10-13.

O’Driscoll, M., G. Ribeiro Dos Santos, L. Wang, D. A. T. Cummings, A. S. Azman, J. Paireau, A. Fontanet, S. Cauchemez, and H. Salje (2020). Age-specific mortality and immunity patterns of sars-cov-2. Nature, 1-6.

Rodríguez, J., M. Patón, and J. M. Acuña (2020). Covid-19 vaccine prioritisation to the groups with the most interactions can substantially reduce total fatalities. medRxiv, 2020.10.12.20211094.

Rosen, S. (1981). Valuing health risk. The American Economic Review 71(2), 241-245.

Selvavinayagam, T., A. Somasundaram, J. M. Selvam, S. Ramachandran, P. Sampath, V. Vijayalakshmi, C. Ajith Brabhu Kumar, S. Subramaniam, S. Raju, R. Avudaiselvi, V. Prakash, N. Yogananth, G. Subramanian, A. Roshini, D. Dhiliban, S. Imad, V. Tandel, R. Parasa, S. Sachdeva, and A. Malani (2021). Seroprevalence in tamil nadu through india's two covid waves: Evidence on antibody decline following infection and vaccination. medRxiv, 2021.11.14.21265758.

Voysey, M., S. A. C. Clemens, S. A. Madhi, L. Y. Weckx, P. M. Folegatti, P. K. Aley, B. Angus, V. L. Baillie, S. L. Barnabas, Q. E. Bhorat, S. Bibi, C. Briner, P. Cicconi, A. M. Collins, R. Colin-Jones, C. L. Cutland, T. C. Darton, K. Dheda, C. J. A. Duncan, K. R. W. Emary, 
K. J. Ewer, L. Fairlie, S. N. Faust, S. Feng, D. M. Ferreira, A. Finn, A. L. Goodman, C. M. Green, C. A. Green, P. T. Heath, C. Hill, H. Hill, I. Hirsch, S. H. C. Hodgson, A. Izu, S. Jackson, D. Jenkin, C. C. D. Joe, S. Kerridge, A. Koen, G. Kwatra, R. Lazarus, A. M. Lawrie, A. Lelliott, V. Libri, P. J. Lillie, R. Mallory, A. V. A. Mendes, E. P. Milan, A. M. Minassian, A. McGregor, H. Morrison, Y. F. Mujadidi, A. Nana, P. J. O'Reilly, S. D. Padayachee, A. Pittella, E. Plested, K. M. Pollock, M. N. Ramasamy, S. Rhead, A. V. Schwarzbold, N. Singh, A. Smith, R. Song, M. D. Snape, E. Sprinz, R. K. Sutherland, R. Tarrant, E. C. Thomson, M. E. Török, M. Toshner, D. P. J. Turner, J. Vekemans, T. L. Villafana, M. E. E. Watson, C. J. Williams, A. D. Douglas, A. V. S. Hill, T. Lambe, S. C. Gilbert, A. J. Pollard, M. Aban, F. Abayomi, K. Abeyskera, J. Aboagye, M. Adam, K. Adams, J. Adamson, Y. A. Adelaja, G. Adewetan, S. Adlou, K. Ahmed, Y. Akhalwaya, S. Akhalwaya, A. Alcock, A. Ali, E. R. Allen, L. Allen, T. C. D. S. C. Almeida, et al. (2021). Safety and efficacy of the chadox1 ncov-19 vaccine (azd1222) against sars-cov-2: an interim analysis of four randomised controlled trials in brazil, south africa, and the uk. The Lancet 397(10269), 99-111.

Worldometers (2021). Covid-19 coronavirus pandemic. Report, Worldometers.info. 


\section{Figures and Tables}

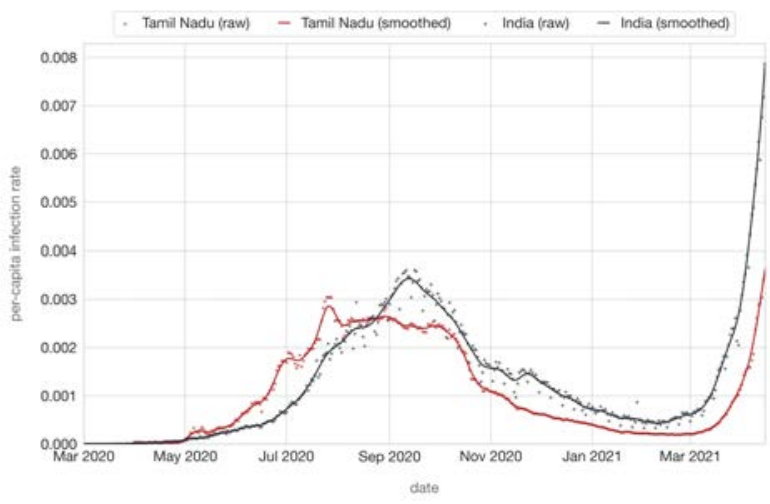

(a) Historic infection rate

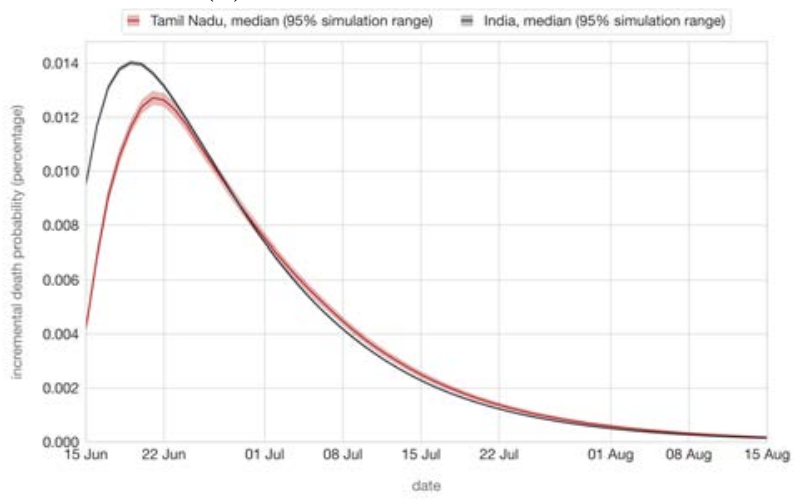

(c) Projected log-probability of death

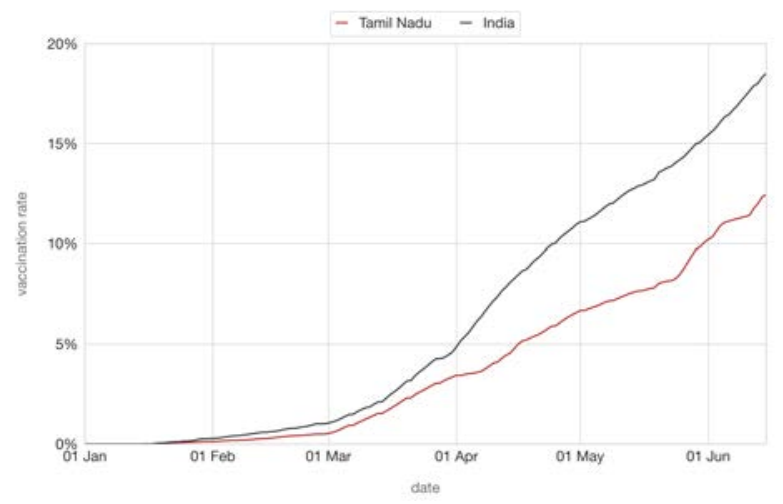

(b) Historic vaccination rate

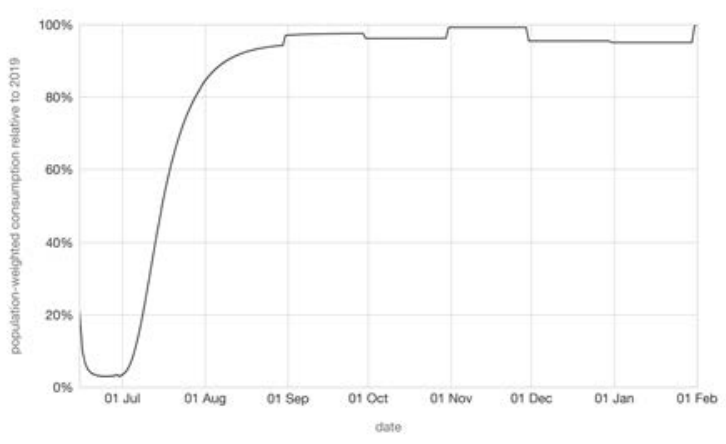

(d) Projected consumption

Figure 1: Past infection rate and reproductive rate and projected death rate and consumption assuming no vaccination in India overall compared to the same in Tamil Nadu.

Note. (A) Points and line report raw and smoothed seroprevalence-scaled infection rate, respectively. (B) Line reports mean reproductive rate and shaded area the 95\% confidence interval. (C) A no-vaccination simulation was run from Jan 01, 2021 for each district in India, and death probabilities were calculated from total deaths across all district-specific models. 


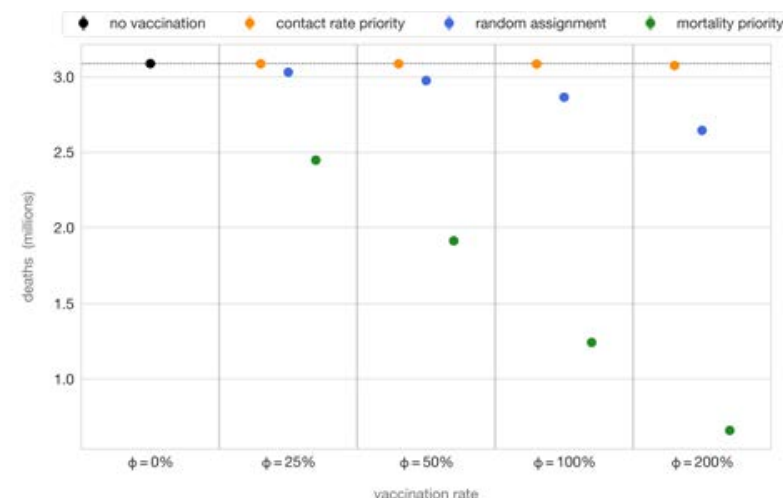

(a) Deaths

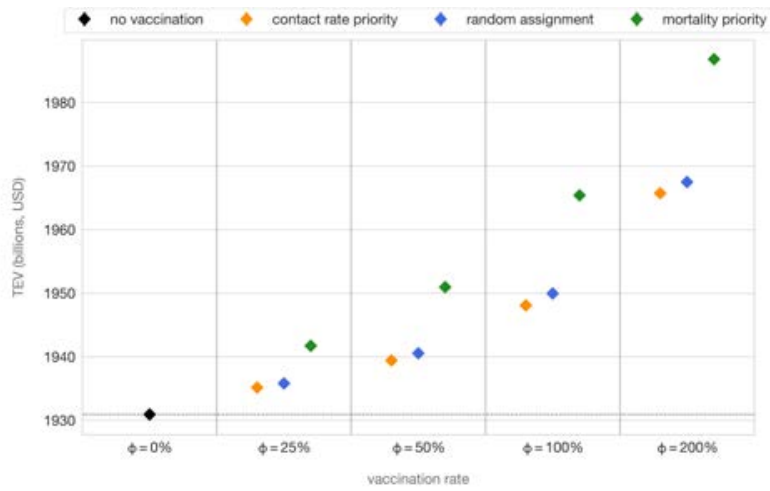

(c) Total economic value (TEV)

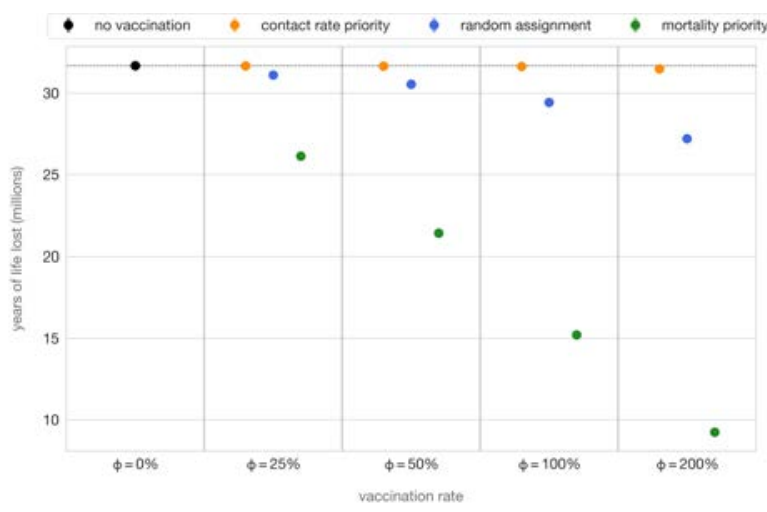

(b) Years of life lost (YLL)

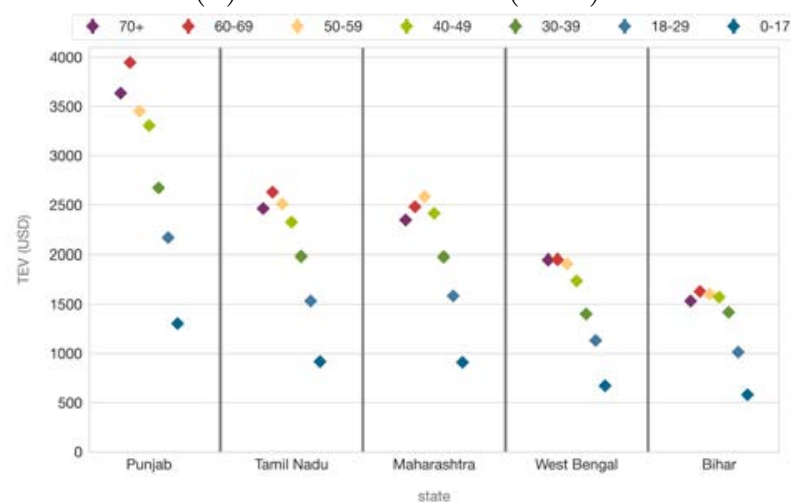

(d) TEV by age group across four states

Figure 2: Value from different vaccination strategies in India.

Note. Each whisker plot gives the median and range of outcomes across 1,000 simulations per vaccination policy. Vaccination rates $(\phi)$ are the fraction of a district vaccinated each year. Vaccine efficacy is assumed to be $70 \%$. Policies simulated include no vaccination $(\phi=0)$ and different prioritization schemes and vaccination rates. 


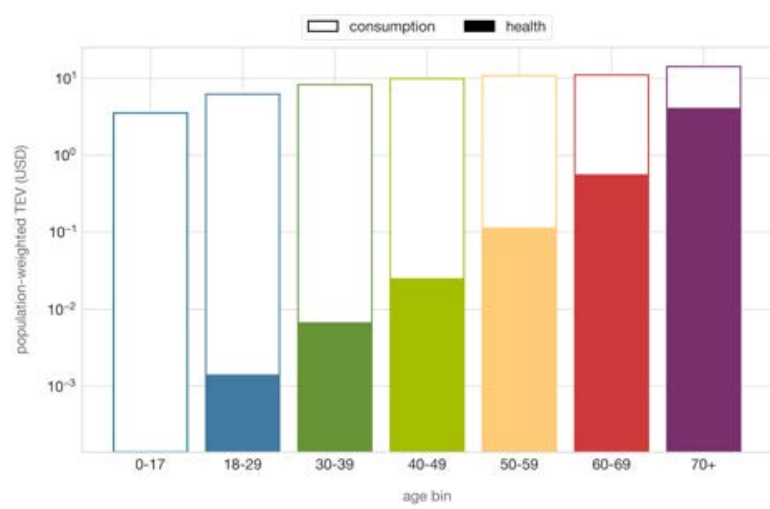

(a) Contribution of health and consumption gains to total economic value
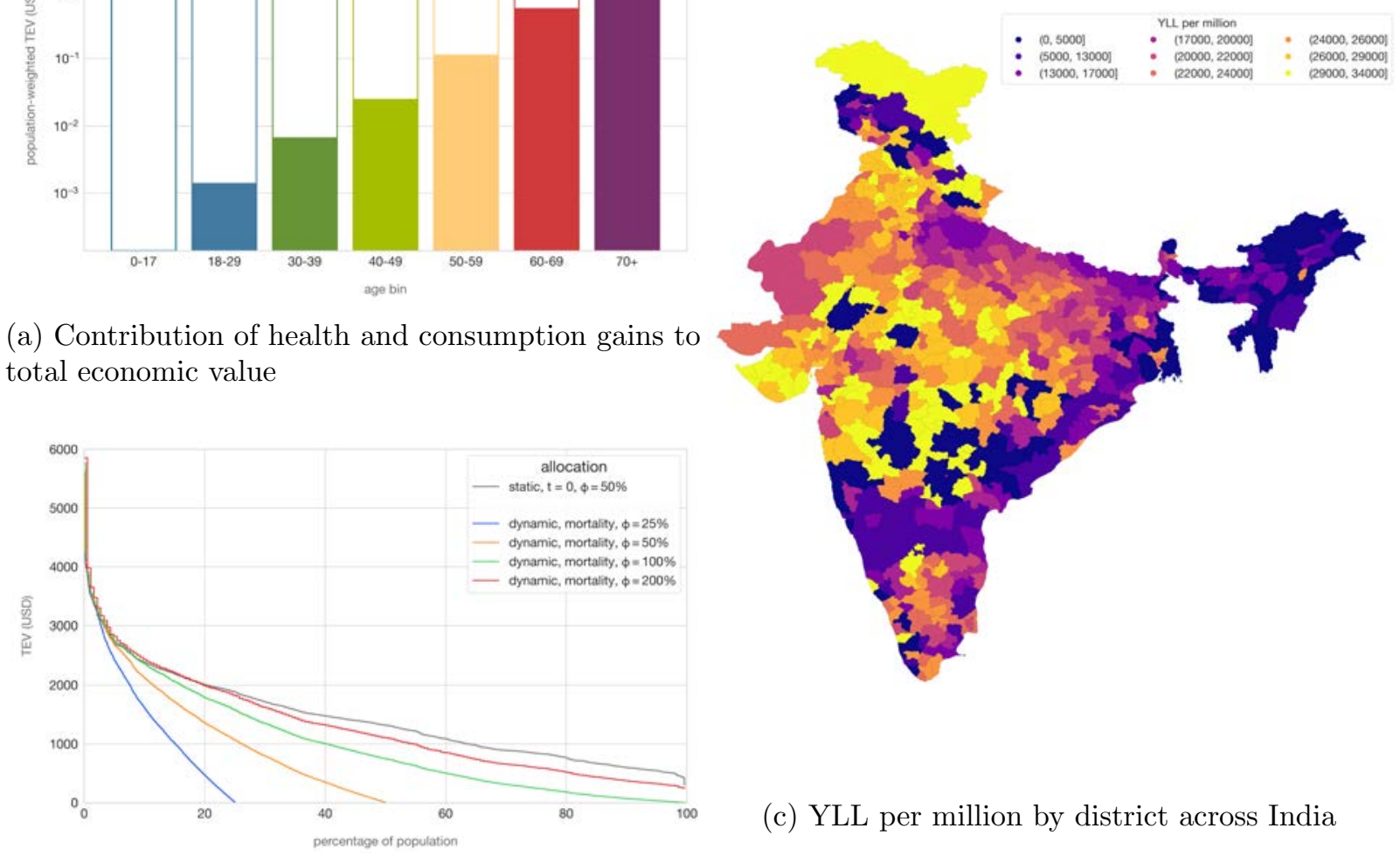

(b) Demand curves for Tamil Nadu

Figure 3: Aspects of value from vaccination across India.

Note. (A) Decomposition of aggregate total economic value at level of age bins into components associated with improved survival probability and with improved consumption. Total economic value calculated using per capita present value of willingness-to-pay for gains in longevity from a random assignment vaccination policy assuming $50 \%$ of the population is vaccinated annually with a $70 \%$ effective vaccine. (B) Dynamic social demand curve for India under a mortality-rate prioritization policy and $50 \%$ vaccination rate per year. (C) Years of life lost per district assuming a random assignment vaccination policy and $50 \%$ vaccination rate. 


\section{Supplementary Online Materials}

\section{A Epidemiological model and survival probability}

We obtain survival probabilities for different age groups $j$ in location $k$ at each day $t$ starting from 15 June 2021, from simulation of a compartmental model for each district $k$. We first simulate a model without vaccination, then simulate the model with vaccination.

\section{A.1 Base model without vaccination}

The basic compartmental model without vaccination for each location is described by the following equations:

$$
\begin{aligned}
\frac{\mathrm{d} S}{\mathrm{~d} t} & =\dot{S}=-\frac{S}{N} \beta I \\
\frac{\mathrm{d} I}{\mathrm{~d} t} & =\dot{I}=\frac{S}{N} \beta I-\gamma I \\
\frac{\mathrm{d} R}{\mathrm{~d} t} & =\dot{R}=(1-\mu) \gamma I \\
\frac{\mathrm{d} D}{\mathrm{~d} t} & =\dot{D}=\mu \gamma I
\end{aligned}
$$

where $S$ is the number of susceptible, $I$ is the number of infectious, $R$ is the number recovered, $D$ is the number deceased, and $N$ is the population size. We suppress subscript $k$ denoting location to simplify the exposition. We have suppressed subscript $j$ because this base model does not have different dynamics by age; rather the model is simulated for the whole population in a location and then compartments are allocated to age bins based on estimated age allocations for certain compartments. The time-step is one day. The key parameters in the base model are the daily contact rate $\beta$, the recovery rate $\gamma$, and the mortality $\mu$. We set $\gamma=0.1$. (90\% of cases are asymptomatic (Kumar et al., 2021) and a meta-analysis of duration of symptomatic and asymptomatic cases (Byrne et al., 2020) suggests a weighted average duration of infectivity of roughly 10 days.) We will obtain $\beta$ from our estimates of $R_{t}$ and estimate $\mu$ from data (Section A.3).

We simulate the basic model as follows. Borrowing from Bettencourt and Soman (2020) and Bettencourt and Ribeiro (2008), we discretize this system of ordinary differential equations, assume the evolution of the infectious time series $I(t)$ is approximated well by a time-dependent branching factor $(I(t+\Delta t)=I(t) \cdot b(t))$, and equate each time step update to the mean of a Poisson draw. At each time step, we then calculate the change in total cases $\Delta T$, net change in infectious $(\Delta I)$, 
recovered $(\Delta R)$, and deceased $(\Delta D)$ for the entire population according to:

$$
\begin{aligned}
& \Delta T(t) \sim \operatorname{POISSON}\left\{\Delta T(t-1) \cdot \exp \left(\gamma\left(R_{t}-1\right)\right)\right\} \\
& \Delta R(t) \sim \operatorname{POISSON}\{(1-\mu) \cdot \gamma \cdot I(t-1)\} \\
& \Delta D(t) \sim \operatorname{POISSON}\{\mu \cdot \gamma \cdot I(t-1)\} \\
& \Delta I(t)=\Delta T(t)-\Delta R(t)-\Delta D(t)
\end{aligned}
$$

The time-dependent reproductive rate $R_{t}$ is estimated from data (Section A.3).

At each timestep, we apportion district-level increments above to age bins indexed by $j$ based on the population shares of the preceding components at the previous timestep:

$$
\begin{gathered}
\Delta I_{j}(t)=\frac{S_{j}(t-1)}{S(t-1)} \cdot \Delta I(t), \\
\Delta R_{j}(t)=\frac{I_{j}(t-1)}{I(t-1)} \cdot \Delta R(t), \\
\Delta D_{j}(t)=\frac{I_{j}(t-1)}{I(t-1)} \cdot \Delta D(t)
\end{gathered}
$$

To initialize the simulation, we apportion based on observed age-specific data (see Section A.3):

$$
\begin{array}{r}
\Delta I_{j}\left(t_{0}\right)=\frac{\hat{R}_{j}+\hat{D}_{j}}{\sum_{j}\left(\hat{R}_{j}+\hat{D}_{j}\right)} \cdot \Delta I\left(t_{0}\right), \\
\Delta R_{j}\left(t_{0}\right)=\frac{\hat{R}_{j}}{\sum_{j} \hat{R}_{j}} \cdot \Delta R\left(t_{0}\right), \\
\Delta D_{j}\left(t_{0}\right)=\frac{\hat{D}_{j}}{\sum_{j} \hat{D}} \cdot \Delta D\left(t_{0}\right)
\end{array}
$$

Because we subdivide district-specific compartments $(I, R, D)$ to age bins ex post, there is is no variation in age shares across districts. Geographic variation in age distributions will implicitly be absorbed by the age distribution among susceptibles, an item that is not critical to the model.

\section{A.2 Data}

We employ three data sets to estimate key parameters of the base model.

Official reports of daily cases and deaths. For analysis of vaccination policy in Tamil Nadu, we employ employ data from the Tamil Nadu Government on daily cases and deaths by district, demographic group and day since the start of the epidemic. For other states, we employ 
data on daily cases and deaths by district and day from www.covid19india.org, a website that scrapes public reports, tradition media and social media for government data on the COVID-19 epidemic.

Seroprevalence in Tamil Nadu. We obtain data from a population-representative seroprevalence survey by the state of Tamil Nadu. The survey was conducted on 26,140 persons from 15 October - 30 November 2020. The sample included individuals aged 18 and above who provided informed consent. Details on the resulting sample and its basic findings are available from Malani et al. (2021). The survey was designed to be representative for urban and rural areas of districts and for demographic groups defined by age and gender.

Indian Census 2011. We employ the Census because it is the latest data for which we have population broken down by location and age.

\section{A.3 Estimation for base model}

We estimate $R_{t}$ from official data on official reports on daily deaths using the parametric system described in Bettencourt and Soman (2020). We have access to district-level reported cases; so our $R_{t}$ estimate is district-specific but not district $\times$ age-specific. We assume that non-reporting of COVID-19 deaths is orthogonal to trends in actual COVID-19 deaths. Moreover, we assume the latter trends are the same for all age groups in a district.

We estimate $R_{j k}$, total number of recovered persons in age group $j$ in district $k$, from the Tamil Nadu sero-survey. Our estimator for $R_{j k}$ is the share of seropositive persons that are in age bin $j$ (in Tamil Nadu sero-data) times the total number of recovered persons $R_{k}$ in district $k$.

$$
R_{j k}=\frac{\mathbb{I}[\text { positive, } \mathrm{TN}, \text { age } j]}{\mathbb{I}[\text { positive }, \mathrm{TN}]} \times R_{k}
$$

$R_{k}$ is estimated directly for Tamil Nadu: it is (a) the ratio of sero-positives in $k$ divided by the number tested in $k$ in the Tamil Nadu survey times (b) the population of $k, N_{k}$. For districts outside Tamil Nadu, it is a weighted average of seroprevalence in urban areas $\left(r_{\text {urban }}^{\mathrm{TN}}\right)$ and in rural areas $\left(r_{\text {rural }}^{\mathrm{TN}}\right)$ of Tamil Nadu, with the weights being the shares of district $k$ (outside Tamil Nadu) that in urban $\left(\alpha_{k, \text { urban }}\right)$ and rural areas $\left(\alpha_{k, \text { rural }}\right)$, according to the 2011 Indian Census and its 
definitions of urban and rural.

$$
\begin{aligned}
& R_{k}^{\mathrm{TN}}=\frac{\mathbb{I}[\text { positive, TN, in } k]}{\mathbb{I}[\text { tested, TN , in } k]} \times N_{k}, \quad r_{\text {urban }}^{\mathrm{TN}}=\frac{\mathbb{I}[\text { positive, TN, urban }]}{\mathbb{I}[\text { tested, TN }, \text { urban }]}, \\
& r_{\text {rural }}^{\mathrm{TN}}=\frac{\mathbb{I}[\text { positive, TN, rural }]}{\mathbb{I}[\text { tested, TN, rural }]}, \quad R_{k}=\left[\alpha_{k, \text { urban }} r_{\text {urban }}^{\mathrm{TN}}+\left(1-\alpha_{k, \text { urban }}\right) r_{\text {rural }}^{\mathrm{TN}}\right] \times N_{k}
\end{aligned}
$$

We use seropositive persons because these are a subset of recovered persons. This is unbiased for population shares for different ages under the assumption that antibody concentrations decline at the same rate for all ages.

We estimate $D_{j k}$ in district $k$ in two steps. First, using official data on cumulative deaths in Tamil Nadu up until 23 December 2020, the last day for which we have daily death data, we estimate $D_{j}^{T N}$, the share of deaths in Tamil Nadu that are in age $j$, with the total share of cumulative deaths reported the state that are in age bin $j$. Second, we assume that the age distribution of deaths in district $k$, regardless of state, is the same as in Tamil Nadu, and we estimate $D_{j k}$ by scaling the Tamil Nadu share in $j$ by $D_{k}$, local deaths in $k$ :

$$
D_{j k}=\frac{\mathbb{I}[\text { dead, TN, age } j]}{\mathbb{I}[\text { dead, TN }]} \times D_{k}
$$

We estimate the overall infection fatality rate $\mu^{T N}$ in Tamil Nadu as the ratio of (a) cumulative reported deaths in official reports until 2 December 2020 (the end of the Tamil Nadu sero-survey plus two days) and (b) the number previously infected based on Tamil Nadu sero-prevalence data, $R^{T N}=N^{T N} \times(\mathbb{I}[$ positive, $\mathrm{TN}] / \mathbb{I}[$ tested, $\mathrm{TN}])$. We apply this same rate to all districts in Tamil Nadu. For districts outside Tamil Nadu, we estimate IFR as the ratio of (a) cumulative deaths until 2 December 2020 to (b)

$$
R_{s}=\left[\alpha_{s, \text { urban }} r_{\text {urban }}^{\mathrm{TN}}+\left(1-\alpha_{s, \text { urban }}\right) r_{\text {rural }}^{\mathrm{TN}}\right] \times N_{s}
$$

where $s$ indexes states.

\section{A.4 Model with vaccination}

To model vaccination, we modify the basic model in (1) - (4) to capture vaccination of the population at rate $\phi \cdot N / 365$ people vaccinated per day. Our compartmental model is described in Figure A1.

We assume vaccination is implemented without regard to a person's immune status, but that 
dead people and people previously vaccinated are not vaccinated. Although the total number of vaccinations handed out each year is $\phi N$, the entire population is not eligible to be vaccinated. Only those who are alive and not previously vaccinated can be vaccinated $(S+I+R)$. Therefore, the compartment level vaccination rate for each eligible compartment is the same and equal to $\psi=\phi N /[365 \cdot(S+I+R)]$. The age composition of who is vaccinated in eligible bins depends on the vaccination policy, so the effective rate of vaccination will vary by age group and compartment.

To capture the consequences of vaccination we introduce 4 additional states: a susceptible person given an effective dose $\left(S_{V M}\right.$, because she is immune), a susceptible person given an ineffective dose $\left(S_{V N}\right.$, because she is not immune), an infected person given an ineffective dose $\left(I_{V N}\right)$, and a recovered person given a dose $\left(R_{V M}\right.$, because she is immune because we assume that being recovered is equivalent to having an effective dose). This is executed separately in each district $k$. Note that transition rates to vaccinated may vary with age and the death states do vary with age.

An effective vaccine is defined as one which protects against death. Vaccine efficacy is given by $v_{e}$, which is the fraction that are protected against death. We implement this by moving a fraction $v_{e}$ of vaccinated individuals to a bin $\left(S_{V M}\right)$ that does not go through an infection, recovery and death process since there is no risk of death and valuation of vaccination only depends on death. Because even ineffective vaccine protects against death, individuals who receive ineffective vaccine are able to be infected $\left(I_{V N}\right)$, but they always recover and never die.

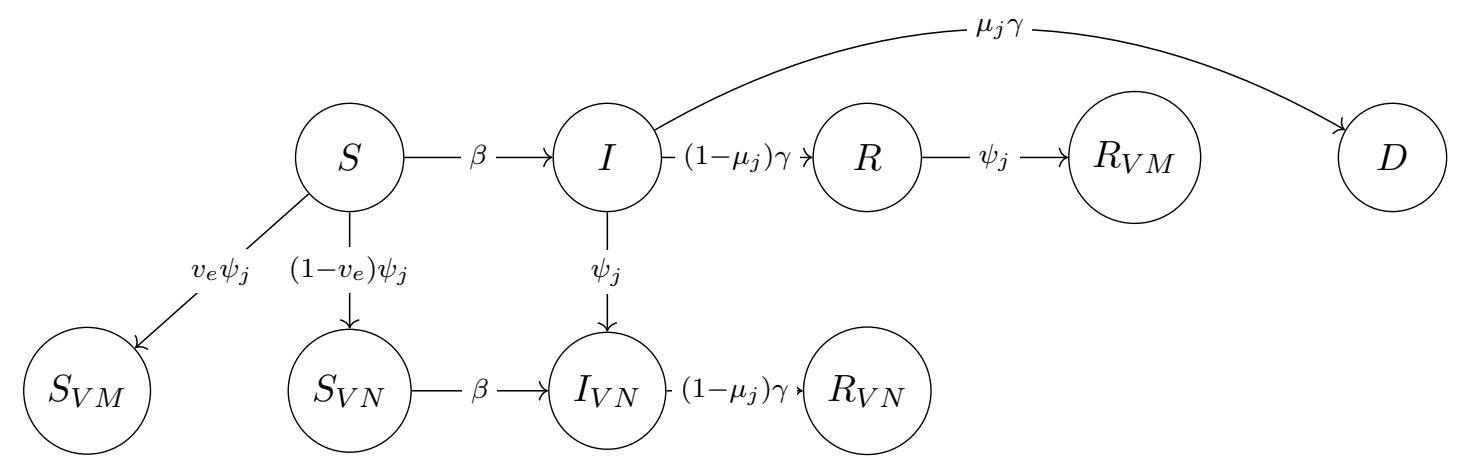

Figure A1: Flow diagram for compartmental model with vaccination 


\begin{tabular}{l|ccc}
\hline Parameter & Symbol & Description & value(s) \\
\hline Reproductive rate & $R_{t}$ & Number of new infections for each existing infection & Estimated \\
Infectious period & $\gamma^{-1}$ & Time period during which patient can infect others & 10 days \\
Mortality (in TN) & $\mu$ & Probability of death given infection & Estimated \\
Vaccination rate & $\phi$ & Fraction of population vaccinated yearly & $\{0.25,0.50,1.0,2.0\}$ \\
Vaccine effectiveness & $v_{e}$ & Probability of vaccine providing immunity & 0.7 \\
\hline
\end{tabular}

Table A1: Table of parameters

\section{A.5 Table of parameters for base and vaccination models}

\section{B Vaccination strategies}

The distribution of vaccine doses over age bins is guided by vaccination strategy. We simulate 6 vaccinations strategies: 3 priority schedules crossed with 2 vaccination rates. The priority schedules are summarized in Table A2, The four vaccination rates are 25\%, 50\%, 100\%, and 200\% of the population of a district per year.

\begin{tabular}{l|lcc}
\hline Strategy & Assignment across districts & Eligible population & Assignment within districts \\
\hline Random assignment & Proportional to population & All living & Random by age \\
Minimize mortality & Proportional to population & All living & Highest age bins first \\
Minimize infection & Proportional to population & All living & Age bins with highest contact rate first \\
\hline
\end{tabular}

Table A2: Vaccination priority schedules

Each strategy allocates vaccinations to districts in proportion to the population of those districts. All living individuals, whether or not they were previously infected, are eligible for vaccination. In the random assignment scheme, vaccines are distributed to age bins in proportion to their share of the population. In the mortality rate prioritization scheme, we vaccine in each state $(S, I, R)$ age bins from highest to lowest age. In the contact rate prioritization scheme, we vaccine in each state $(S, I, R)$ age bins from those with highest contact rate to lowest contact rate, where the contact rate is obtained from a large $(N \approx 500,000)$ data set on contact-tracing results in Andhra Pradesh and Tamil Nadu (Laxminarayan et al., 2020). We end the simulation after 1 year has elapsed.

\section{Simulations}

Note that our model without vaccination is identical to our model with vaccination when we set $\phi=0$. Therefore, it is sufficient that we describe our simulation of our vaccination model.

For each time-step $t$, we run the epidemiological model forward according to the update equa- 
tions (5) - (8). Our last estimate of $R_{t}$ from 15 April, 2021, is our estimate for $R_{t}$ going forward. Figure A2a gives the distribution of $R_{t}$ on that date across all the districts of India, with the mean for the five states that we study highlighted. Then, we model the effect of the vaccination scheme in question by removing members of the $S, I$, and $R$ components in accordance with the overall vaccination rate $\phi \cdot N / 365$ (measured in the number of persons per day), and the vaccine effectiveness $v_{e}$.

By iterating this two-phase procedure forward in time, we obtain standard compartment counts for each of the compartments in Figure A1. In addition, we generate three time series. One is the number of people that were vaccinated $\left(N_{V, j k t}^{p}\right)$ and the number not vaccinated $\left(N_{N V, j k t}^{p}\right)$ by date $t$, where both numbers include persons that may be dead by $t$. The second is the probability of vaccination prior to that period $\left(\pi_{j k t}^{p}\right)$ given membership in the alive population at the start of the simulation. The third is the expected probability of survival given that a person is vaccinated $\left(q_{j k t}^{p}\left(v_{i j k t}^{p}=1\right)\right)$ or not $\left(q_{j k t}^{p}\left(v_{i j k t}^{p}=0\right)\right)$, where $v_{i j k t}^{p}$ is an indicator for whether a person was vaccinated by time period $t$. These are defined as follows

$$
\begin{array}{rl}
N_{V, j k t}^{p} & =S_{V M, j k t}^{p}+S_{V N, j k t}^{p}+I_{V N, j k t}^{p}+D_{V N, j k t}^{p}+R_{V N, j k t}^{p}+R_{V M, j k t}^{p}, \\
N_{N V, j k t}^{p} & =N_{j k}^{p}-N_{V, j k t}^{p} \\
\pi_{j k t}^{p} & =\frac{N_{V, j k t}^{p}}{N_{j k}^{p}}, \\
q_{j k t}^{p}\left(v_{j k t}^{p}=1\right) & =\frac{N_{V, j k t}^{p}-D_{V N, j k t}^{p}}{N_{V, j k t}^{p}}, \\
q_{j k t}^{p}\left(v_{j k t}^{p}=0\right) & =\frac{N_{N V, j k t}^{p}-\left(D_{j k t}^{p}-D_{j k 0}\right)}{N_{N V, j k t}^{p}} \\
q_{j k t}^{p}\left(v_{j k t}^{p}=1\right) & =1-\frac{D_{V N, j k t}^{p}-D_{V N, j k, t-1}^{p}}{N_{V, j k t}^{p}} \\
N_{V, j k 0}^{p}>0 & 0 \\
q_{j k t}^{p}\left(v_{j k t}^{p}=0\right) & =1-\frac{D_{V N, j k 0}^{p}=0}{N_{N V, j k t}^{p}}
\end{array}
$$

We run 1000 simulations for each policy in each location in India, where a policy is defined by a vaccine (defined by efficacy $v_{e}$ ), a vaccination rate per year (defined as fraction $\phi$ vaccinated per year), and a prioritization schedule and a location is defined as a district. 


\section{Evaluating vaccination strategies}

We evaluate each of the 6 vaccination strategies against a counterfactual of no vaccination and existing contact rates. We measure value in 5 ways.

1. Number of lives saved

2. Number of discounted life-years saved

3. The value of lives saved using a static value of a statistical life $\left(\mathrm{VSL}_{k}\right)$

4. The value of life years saved using a static value of a statistical life year $\left(\mathrm{VSLY}_{k}\right)$

5. The value of life years using willingness to pay in an economic framework

The first two approaches are obtained directly from the compartmental models above. We explain methods 3 and 4 in section $\mathrm{G}$. We explain the last method in section $\mathrm{H}$. However, to explain the methods for 3 - 5, we need to present our economic framework (section E) and how we project consumption (section F).

\section{E Economic framework}

We build our economic framework (Murphy and Topel, 2006) starting with the remaining lifetime utility of an individual at time $t$ with with attributes $\mathbf{z}$ (including age $a$, gender and location):

$$
U(\mathbf{x}, t)=\int_{t}^{t+(T-a)} e^{-\rho(s-t)} q(s, \mathbf{z}) u(c(s, \mathbf{z})) \mathrm{d} s
$$

where $T$ is life expectancy, $\rho$ is a discount rate, $q(s, \mathbf{z})$ is the survival probability for a person of age $a$ at time $s$, and $c(s, \mathbf{z})$ is consumption at time $s$.

We define the total economic value conveyed to a person of a given policy ( $p$ ) to be the individual willingness to pay for longevity and consumption equivalent to that achieved under $\mathrm{p}$. The individual willingness to pay for this change is typically expressed (Rosen, 1981; Lakdawalla and Phelps, 2020; Becker et al., 2005; Garber and Phelps, 1997) as

$$
W^{p}(t, \mathbf{z})=\delta \int_{t}^{\infty} e^{-\rho(s-t)} \cdot q^{p}(s, \mathbf{z}) c^{p}(s, \mathbf{z}) \mathrm{d} s
$$


where $\delta$ is typically a multiple between 1 and 3 (Lakdawalla and Phelps, 2020). In our calculations we set $\delta=1$. We employ a discrete time version of this last equation, where time units are days.

We define the incremental total economic value of one vaccination policy ( $\mathrm{p}$ ) over a no vaccination policy (0) as equal to the economic value of $\mathrm{p}$ relative to 0 :

$$
W^{p}(t, \mathbf{z})-W^{0}(t, \mathbf{z})
$$

We define the per capita total economic value of a given policy to be the average individual willingness to pay for the policy over some population, e.g., an age group in a location. We define the aggregate total economic value of a policy to be the sum of value across the overall population in a location. We define the per capita and aggregate incremental total economic value in an analogous manner.

\section{F Consumption}

\section{F.1 Data and estimation}

Data on consumption are obtained from the Center for Monitoring Indian Economy's Consumer Pyramids Household Survey (CPHS, https://consumerpyramidsdx.cmie.com/). This is a nationwide, population-representative panel data set on $\sim 174,000$ households. Each household is surveyed every 4 months; because sampling is continual, the survey reports financial indicators for $1 / 4$ of households each month. We employ data from January 2018 to March 2021. The data are representative at the level of homogeneous regions (HR), defined as clusters of economically and demographically-similar districts within a state; we estimate parameters at the HR level and apply those estimates to each district in the HR.

We allocate household consumption to individual members using the OECD's modified scale (Hagenaars et al. 1994). We estimate mean consumption by age group and homogeneous region in each month; we assume group means are the for each district within a homogeneous region.

\section{F.2 Forecasting consumption}

In order to forecast consumption, we take two steps. First, we estimate a linear regression of past consumption on local disease conditions. Our goal is to be able to take projections on those conditions from our epidemiological model in section $\mathrm{A}$ and predict consumption. Our regression 
specification, given below, is intended to generate a return to 2019 economic conditions when new infections and deaths are zero.

$$
r c_{k, t}=f\left(I_{k, t}, D_{k, t}, k, t\right)=\theta_{0}+\psi_{k}+\delta_{t}+\theta_{I} I_{k, t}+\theta_{D} D_{k, t}+e_{k, t}
$$

where $c_{k, t}$ is mean per capita household consumption in district $k$ and $r c_{k, t}=\left(c_{d, k, t}-c_{k, 2019}\right) / c_{k, 2019}$ is decline in mean household consumption relative to the average consumption in 2019 in district $k$ on day $t$. We include a location $\psi_{k}$ and a month-of-year fixed effect $\delta_{t}$. We do not include a month $\times$ year fixed effect because we will use this regression model to project income in the future, and we cannot project what future time $\times$ year fixed effects will be. The error term is $e_{k, t}$. $I_{k, t}$ is new COVID-19 infections per capita for district $k$ on day $t$, and $D_{k, t}$ is new COVID-19 deaths per capita for district $k$ and day $t$. We do not include government orders because our goals is not to casually distinguish voluntary behavioral response to behavioral response to policy; our goal is merely to project consumption with epidemiological data.

We estimate this regression using data on household consumption from the CPHS and on cases and deaths from official reports obtained via the covid19India API. Consumption data is available at the household $\times$ month level for roughly $1 / 4$ of households. We obtain daily consumption data on each household by dividing monthly consumption by the number of days in each month. We compiled a 2011 Census district to 2020 district map to map official case and death data to 2011 districts. Daily cases and deaths are calculated from the number of new daily cases and deaths in a district divided by district population according to the 2011 Indian Census.

Second, we project out consumption under the assumption that vaccination has two effects. One is that a higher vaccination rate increase consumption regardless of whether one is vaccinated. That increase is a function of how vaccination policy affects the population-level trajectory of the epidemic, as estimated in the first step of our forecasting.

The other effect is that vaccination immediately increases the income of the person who is actually vaccinated to the level she enjoyed before the epidemic:

$$
\begin{aligned}
& c_{i j k, t+\tau}^{p}(v=1)=c_{j k, 2019}(1+\hat{r} c(0,0, k, t+\tau)) \\
& c_{i j k, t+\tau}^{p}(v=0)=c_{j k, 2019}\left(1+\hat{r} c\left(I_{k, t+\tau}^{p}, D_{k, t \tau}^{p}, k, t+\tau\right)\right)
\end{aligned}
$$

where $I_{k, t+\tau}$ and $D_{k, t+\tau}$ are projected from simulation of our compartmental model and $\hat{r c}$ is a 
prediction based on estimation of $(26)$. After this is estimated for each household, we allocate consumption to individual members using the OECD modified scale.

\section{G Monetized longevity benefits}

\section{G.1 Value of statistical life}

The value of statistical life (VSL) approach to evaluating vaccination policy $p$ differs from total economic value in two ways. First, it assumes that vaccination does not change consumption. This means that the value of life is calculated as willingness to pay for life at $t=0$, the day before vaccination starts, under the no vaccination policy. TEV allows that vaccination increases consumption thus the willingness to pay for longevity gains equivalent to those under a vaccination policy. Second, it assumes all lives are worth the same, regardless of the number of life years left.

The VSL evaluation of a policy is obtained by multiplying the first approach to valuation vaccination policies (lives saved) in section $\mathrm{D}$ by an estimate of VSL:

$$
V_{k t}^{p, V S L}=L S_{k t}^{p} \cdot V S L_{k t}
$$

where $L S$ is lives saved. We calculate the value of a statistical life in location $k$ with the following formula:

$$
V S L_{k t}=\delta \sum_{j} \frac{N_{j k}}{N_{k}}\left(\sum_{s=0}^{L E_{j}} \beta^{s} E_{\zeta}\left[\hat{c}_{\zeta j k, t+s}^{0}\right]\right)
$$

where $L E_{j}$ is remaining life expectancy in age group $j$ in the absence of COVID-19 (based on Indian Census tables), $\beta$ is the discretized discount factor, which we derive from the risk-free interest rate in India $(4.25 \%), \zeta$ indexes a simulation done for a given location and policy, and $E_{\zeta}[z]$ is the expected value over simulations for a given location and policy.

\section{G.2 Value of statistical life-year}

We calculate the VSLY-based per capita value from a vaccination policy $p$ by age group $j$ in an area $k$ by asking how much individuals are willing to pay to obtain an increase in longevity equivalent to the gain they would experience under that policy under the assumption that vaccination policy does not increase consumption. These calculations are discrete time analogues of (25) with the 
assumption implemented as a restriction on consumption:

$$
V_{i j k t}^{p, V S L Y}=V_{i j k t}^{p}-V_{i j k t}^{0}
$$

where

$$
\begin{aligned}
& V_{i j k t}^{p}=\delta \sum_{s=t}^{\infty} \beta^{s-t}\left[\left(1-\pi_{i j k s}\right) \hat{q}_{i j k s}^{p}(v=0)+\pi_{i j k s}^{p} \hat{q}_{i j k s}^{p}(v=1)\right] E_{\zeta}\left[\hat{c}_{i j k s}^{0}\right], \\
& V_{i j k t}^{0}=\delta \sum_{s=t}^{\infty} \beta^{s-t} \hat{q}_{i j k s}^{0} E_{\zeta}\left[\hat{c}_{i j k s}^{0}\right]
\end{aligned}
$$

To calculate the aggregate social value of vaccinating a group $j$ in an area $k$ or of vaccinating an entire area $k$, we scale up by the relevant population:

$$
V_{j k t}^{p, V S L Y}=N_{j k} V_{i j k t}^{p, V S L Y}, \quad V_{k t}^{p, V S L Y}=\sum_{j} N_{j k} V_{i j k t}^{p, V S L Y}
$$

Population estimates are obtained from the 2011 Indian Census.

\section{H Total economic value of vaccination}

We calculate the per capita total economic value (TEV) from a vaccination policy $p$ by age group $j$ in an area $k$ by asking how much individuals are willing to pay to obtain longevity equivalent to the gain they would experience under that policy. These calculations are discrete time analogues of $(25)$ :

$$
\begin{aligned}
& W T P_{i j k t}^{p}=\delta \sum_{s=t}^{\infty} \beta^{s-t}\left[\left(1-\pi_{i j k t}^{p}\right) \hat{q}_{i j k t}^{p}(v=0) \hat{c}_{j k t}^{p}(v=0)+\pi_{i j k t}^{p} \hat{q}_{i j k t}^{p}(v=1) \hat{c}_{i j k t}^{p}(v=1)\right] \\
& W T P_{i j k t}^{0}=\delta \sum_{s=t}^{\infty} \hat{q}_{i j k t}^{0} \hat{c}_{i j k t}^{0}
\end{aligned}
$$

where policy $p=0$ is the no vaccination policy, $\pi_{i j k t}^{p}$ is the probability that a person is vaccinated by time $t$ under policy $p, \hat{q}_{i j k t}^{p}(v=1)$ is the projected probability of surviving until $t$ under policy $p$ given that one is vaccinated by date $t, \hat{q}_{i j k t}^{p}(v=0)$ is the projected probability of surviving until $t$ under policy $p$ given that one is vaccinated by date $t$, and $\hat{c}_{j k t}^{p}(v)$ is the projected consumption given vaccination status $v \in\{0,1\}$. We define the incremental total economic value of a policy 
$p \neq 0$ as the incremental value over a no vaccination policy:

$$
\Delta W T P_{i j k t}^{p}=W T P_{j k t}^{p}-W T P_{i j k t}^{0}
$$

To calculate the aggregate total economic value of vaccinating a group $j$ in an area $k$ or of vaccinating an entire area $k$, we scale up by population:

$$
\begin{aligned}
\Delta W T P_{j k}^{p} & =N_{j k}\left(W T P_{i j k}^{p}-W T P_{i j k}^{0}\right), \quad \Delta W T P_{k}^{p}=\sum_{j} N_{j k}\left(W T P_{i j k}^{p}-W T P_{i j k}^{0}\right), \\
\Delta W T P^{p} & =\sum_{k} \sum_{j} N_{j k}\left(W T P_{i j k}^{p}-W T P_{i j k}^{0}\right)
\end{aligned}
$$

We divide by the relevant population to get averages.

The critical difference between a VSLY-based and the WTP-based total economic evaluation of vaccination policy is that the latter allows consumption to change with vaccination and values that change in consumption. We decompose the total economic value for a vaccination policy into the component due to improvements in health and the component due to increased consumption:

$$
\Delta W T P_{i j k t}^{p}=\Delta W T P_{i j k t}^{p, \text { health }}+\Delta W T P_{i j k t}^{p, \text { consumption }}
$$

where

$$
\begin{aligned}
\Delta W T P_{i j k t}^{p, \text { health }} & =\delta \sum_{s=t}^{\infty} \beta^{s-t}\left[\left(1-\pi_{i j k t}^{p}\right)\left[\hat{q}_{i j k t}^{p}(v=0)-E_{\zeta}\left[\hat{q}_{i j k t}^{0}\right]\right] \hat{c}_{j k t}^{p}(v=0)\right. \\
& \left.+\pi_{i j k t}^{p}\left[\hat{q}_{i j k t}^{p}(v=1)-E_{\zeta}\left[\hat{q}_{i j k t}^{0}\right]\right] \hat{c}_{i j k t}^{p}(v=1)\right] \\
\Delta W T P_{i j k t}^{p, \text { consumption }} & =\delta \sum_{s=t}^{\infty} \beta^{s-t}\left[\left(1-\pi_{i j k t}^{p}\right) E_{\zeta}\left[\hat{q}_{i j k t}^{0}\right]\left[\hat{c}_{j k t}^{p}(v=0)-E_{\zeta}\left[\hat{c}_{i j k t}^{0}\right]\right]\right. \\
& \left.+\pi_{i j k t}^{p} E_{\zeta}\left[\hat{q}_{i j k t}^{0}\right]\left[\hat{c}_{i j k t}^{p}(v=1)-E_{\zeta}\left[\hat{c}_{i j k t}^{0}\right]\right]\right]
\end{aligned}
$$

This decomposition is written at the individual level. However, we plot the state average.

$$
\begin{aligned}
\Delta W T P^{p, \text { health }} & =\frac{\sum_{k} N_{j k} \Delta W T P_{i j k}^{p, \text { health }}}{\sum_{k} N_{j k}}, \\
\Delta W T P^{p, \text { consumption }} & =\frac{\sum_{k} N_{j k} \Delta W T P_{i j k}^{p, \text { consumption }}}{\sum_{k} N_{j k}}
\end{aligned}
$$


Note that we take expectations over simulations of the no vaccination policy in the calculation above. This is because it is not possible to match each simulation of a policy $\mathrm{p}$ with a simulation of no vaccination. To address this, take three steps. First, we calculate average survival probabilities and consumption across simulations of the no vaccination policy to obtain $\left(E_{\zeta}\left[\hat{q}_{i j k t}^{0}\right], E_{\zeta}\left[\hat{c}_{i j k t}^{0}\right]\right)$. Sec-

ond, we plug these expected values in to the $\left(\Delta W T P_{i j k t}^{p, \text { health }}, \Delta W T P_{i j k t}^{p, \text { consumption }}\right)$ equation above, which are defined at the per-simulation level for policy p. Third, we take averages across policy p simulations of the equations above.

\section{Prioritization}

When calculating priority lists, we fix the vaccination policy to be random assignment. Random assignment implies no priority. By calculating priority from this benchmark we obtain something akin to the incremental gain to switching to a different (non-random assignment) prioritization.

There are two alternatives to a random-assignment benchmark that we do not employ. One is to calculate priorities conditional on mortality or contact rate prioritization. If those yield priorities that are consistent with the stated priorities, one can be more confident that they are internally consistent. One can then choose between them based on which generates more overall value. Second, one can solve a dynamic programming problem where one chooses the optimal priority knowing that changing priorities may change the incremental benefit from vaccinating different groups and thus the optimal priorities, requiring repeated readjustment until one obtains the optimal schedule. While these approaches have value, they add more complication than is warranted in this one paper.

We generate priority lists under our random assignment assumption by calculating the per capita willingness to pay for a longevity improvement equivalent to that achieved under random assignment policy separately for each age and location group using the method explained above, except we restrict our averages to those age and location groups; see Tables A3, A4, A5, A6, and A7 for the top 25 district-age bin combinations by economic value for the states of Tamil Nadu, Punjab, Maharashtra, West Bengal, and Bihar.

\section{J Social demand curve}

We seek to estimate the total number of doses that it would be cost effective for a government to purchase given a vaccination policy. In other words, we want to generate a social demand function that maps number of doses onto per capita social value. When one plugs in a cost per dose, one 
can read off the number of doses that one can purchase before the incremental value of those doses fall below the price of those doses.

The challenge to drawing such a curve is that it is dynamic, i.e., the total economic value curve that describes the value of vaccinating different people in the population is changing as the vaccination campaign unfolds. If one simply maps the $t=0$ value curve, it will not capture actual value unless all individuals are vaccinated immediately. If only a fraction of people are vaccinated each day, there are two countervailing effects on the value curve. On the one hand, the risk of infection is falling, which pushes down valuations. On the other hand, consumption may be rising due to lower infection risk, which pushes up valuations.

We address this problem in two steps. First, for each day we estimate the total economic value of vaccinating each non-vaccinated person given a vaccination policy (e.g., mortality prioritization). This is the total economic value of vaccinating each non-vaccinated person given their age and location. The social demand curve can be obtained by sorting those valuations from highest to lowest and then drawing out the valuations for each person.

Of course, not all people will be vaccinated under a given policy. Indeed, no policy even vaccinates all the highest value persons with the vaccines given each day because geographic allocation is pro rata based on population rather than on total economic value. Therefore, we only draw the social demand curve for the first day of a vaccination drive. This illustrates the potential gain under a vaccination policy. It is also the dynamic social demand curve if the vaccination rate were infinite $(\phi=\infty)$ and everyone were vaccinated immediately.

Second, we plot the average per capita social value generated for the individuals actually selected for vaccination each day under the designated vaccination policy. The persons selected may differ across simulations so we take the average across simulations after we take averages across individuals vaccinated within a simulation:

$$
D_{t}^{p}=E_{\zeta}\left[\frac{\sum_{i j k} W T P_{i j k t, \zeta}^{p} \mathrm{I}\left(v_{i j k t, \zeta}=1\right)}{\sum_{i j k} \mathrm{I}\left(v_{i j k t, \zeta}=1\right)}\right]
$$

Our dynamic demand curve for a given policy is the plot of $D_{t}$ over time.

\section{K Supplementary tables and figures}




\section{Tables}

\section{K.0.1 Priority lists}

Tamil Nadu

\begin{tabular}{llr}
\hline district & age bin & per capita TEV (USD) \\
\hline Chennai & $70+$ & 3540.65 \\
Chennai & $60-69$ & 3365.70 \\
Chennai & $50-59$ & 3259.90 \\
Chennai & $40-49$ & 3096.76 \\
Chengalpattu & $50-59$ & 3091.05 \\
Thiruvallur & $70+$ & 3065.69 \\
Chengalpattu & $60-69$ & 3062.88 \\
Thiruvallur & $50-59$ & 3060.01 \\
Chengalpattu & $70+$ & 3037.94 \\
Kancheepuram & $70+$ & 3036.78 \\
Thiruvallur & $60-69$ & 3031.20 \\
Kancheepuram & $50-59$ & 3010.99 \\
Kancheepuram & $60-69$ & 2986.51 \\
Ranipet & $50-59$ & 2947.62 \\
Tirupathur & $50-59$ & 2923.77 \\
Vellore & $50-59$ & 2909.53 \\
Chengalpattu & $40-49$ & 2850.55 \\
Ranipet & $60-69$ & 2834.20 \\
Thiruvallur & $40-49$ & 2827.37 \\
Tirupathur & $60-69$ & 2817.87 \\
Vellore & $60-69$ & 2799.09 \\
Kallakurichi & $60-69$ & 2778.64 \\
Kancheepuram & $40-49$ & 2777.11 \\
Tirupathur & $70+$ & 2742.02 \\
Tiruvannamalai & $60-69$ & 2735.29 \\
\hline & &
\end{tabular}

Table A3 
Punjab

\begin{tabular}{llr}
\hline district & age bin & per capita TEV (USD) \\
\hline S.A.S. Nagar & $70+$ & 4227.12 \\
S.A.S. Nagar & $60-69$ & 4225.91 \\
Shahid Bhagat Singh Nagar & $70+$ & 4171.79 \\
Rupnagar & $70+$ & 4160.34 \\
Hoshiarpur & $70+$ & 4147.45 \\
Gurdaspur & $70+$ & 4092.15 \\
Barnala & $60-69$ & 4091.19 \\
Pathankot & $70+$ & 4077.57 \\
Kapurthala & $60-69$ & 4070.03 \\
Ludhiana & $60-69$ & 4063.36 \\
Amritsar & $60-69$ & 4061.13 \\
Jalandhar & $60-69$ & 4037.71 \\
Patiala & $60-69$ & 3993.60 \\
Fatehgarh Sahib & $60-69$ & 3985.64 \\
Faridkot & $60-69$ & 3957.68 \\
Bathinda & $60-69$ & 3924.07 \\
Sangrur & $60-69$ & 3923.74 \\
Tarn Taran & $60-69$ & 3919.57 \\
Pathankot & $60-69$ & 3913.69 \\
Sri Muktsar Sahib & $60-69$ & 3906.62 \\
Moga & $60-69$ & 3902.56 \\
Fazilka & $60-69$ & 3902.02 \\
Gurdaspur & $60-69$ & 3889.88 \\
Barnala & $70+$ & 3883.53 \\
Ferozepur & $60-69$ & 3881.58 \\
\hline
\end{tabular}

Table A4 
Maharashtra

\begin{tabular}{llr}
\hline district & age bin & per capita TEV (USD) \\
\hline Mumbai & $70+$ & 4780.10 \\
Mumbai & $60-69$ & 4696.15 \\
Thane & $70+$ & 4647.24 \\
Thane & $60-69$ & 4261.98 \\
Palghar & $70+$ & 4225.33 \\
Mumbai & $50-59$ & 4218.14 \\
Thane & $50-59$ & 4013.55 \\
Palghar & $60-69$ & 3897.48 \\
Mumbai & $40-49$ & 3847.73 \\
Palghar & $50-59$ & 3677.96 \\
Thane & $40-49$ & 3649.55 \\
Palghar & $40-49$ & 3346.69 \\
Aurangabad & $60-69$ & 3112.36 \\
Aurangabad & $50-59$ & 3107.64 \\
Thane & $30-39$ & 3101.14 \\
Aurangabad & $40-49$ & 3068.14 \\
Aurangabad & $70+$ & 3017.71 \\
Mumbai & $30-39$ & 3006.65 \\
Nagpur & $60-69$ & 2888.99 \\
Nagpur & $70+$ & 2858.32 \\
Palghar & $30-39$ & 2844.51 \\
Pune & $50-59$ & 2804.48 \\
Raigad & $70+$ & 2771.62 \\
Nagpur & $50-59$ & 2741.12 \\
Pune & $70+$ & 2719.44 \\
\hline
\end{tabular}

Table A5 
West Bengal

\begin{tabular}{llr}
\hline district & age bin & per capita TEV (USD) \\
\hline Kolkata & $70+$ & 3321.68 \\
North 24 Parganas & $70+$ & 3105.59 \\
Howrah & $70+$ & 3004.53 \\
Kolkata & $60-69$ & 2952.52 \\
North 24 Parganas & $60-69$ & 2798.24 \\
Kolkata & $50-59$ & 2796.07 \\
South 24 Parganas & $60-69$ & 2711.61 \\
Howrah & $60-69$ & 2703.76 \\
Howrah & $50-59$ & 2640.97 \\
South 24 Parganas & $70+$ & 2607.48 \\
North 24 Parganas & $50-59$ & 2564.20 \\
South 24 Parganas & $50-59$ & 2534.06 \\
Kolkata & $40-49$ & 2500.90 \\
Howrah & $40-49$ & 2388.01 \\
South 24 Parganas & $40-49$ & 2303.47 \\
North 24 Parganas & $40-49$ & 2257.13 \\
Paschim Bardhaman & $70+$ & 2043.16 \\
Hooghly & $70+$ & 2036.72 \\
Purba Bardhaman & $70+$ & 2022.45 \\
Hooghly & $60-69$ & 1975.76 \\
Paschim Bardhaman & $60-69$ & 1972.55 \\
Hooghly & $50-59$ & 1960.38 \\
Kolkata & $30-39$ & 1959.17 \\
Paschim Bardhaman & $50-59$ & 1958.26 \\
Purba Bardhaman & $60-69$ & 1950.47 \\
\hline
\end{tabular}

Table A6 
Bihar

\begin{tabular}{llr}
\hline district & age bin & per capita TEV (USD) \\
\hline Sheikhpura & $60-69$ & 2045.65 \\
Nalanda & $60-69$ & 2028.17 \\
Nawada & $60-69$ & 2021.86 \\
Gaya & $60-69$ & 2020.15 \\
Lakhisarai & $60-69$ & 2019.18 \\
Jamui & $60-69$ & 2014.73 \\
Banka & $60-69$ & 1982.94 \\
Kishanganj & $70+$ & 1859.98 \\
Sheikhpura & $50-59$ & 1853.70 \\
Purnia & $70+$ & 1852.81 \\
Lakhisarai & $70+$ & 1845.90 \\
Katihar & $70+$ & 1845.74 \\
Nalanda & $50-59$ & 1842.74 \\
Araria & $70+$ & 1841.29 \\
Gaya & $50-59$ & 1832.17 \\
Sheikhpura & $70+$ & 1823.94 \\
Lakhisarai & $50-59$ & 1823.28 \\
Nawada & $50-59$ & 1822.33 \\
Banka & $70+$ & 1818.32 \\
Gaya & $70+$ & 1816.65 \\
Jamui & $50-59$ & 1815.35 \\
Nawada & $70+$ & 1814.15 \\
Nalanda & $70+$ & 1811.85 \\
Jamui & $70+$ & 1807.73 \\
Patna & $50-59$ & 1807.12 \\
\hline
\end{tabular}

Table A7 


\section{Figures}

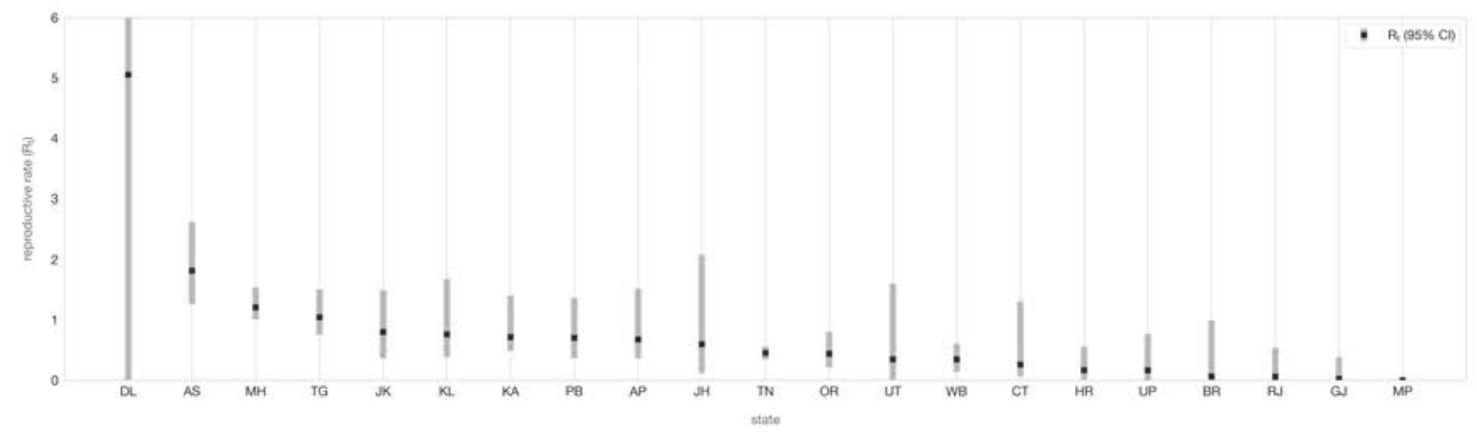

(a) Distribution of $R_{t}$ for states and union territories with population greater than 10 million on last day before simulation begins

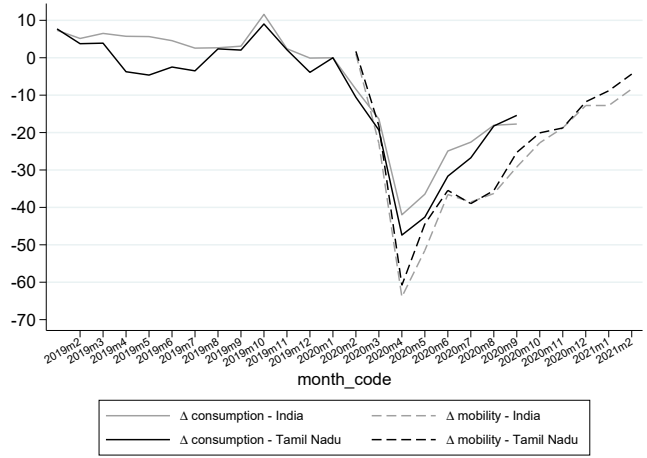

(b) Actual relative consumption and mobility in (c) Projected probability of death, by age, in Tamil Tamil Nadu

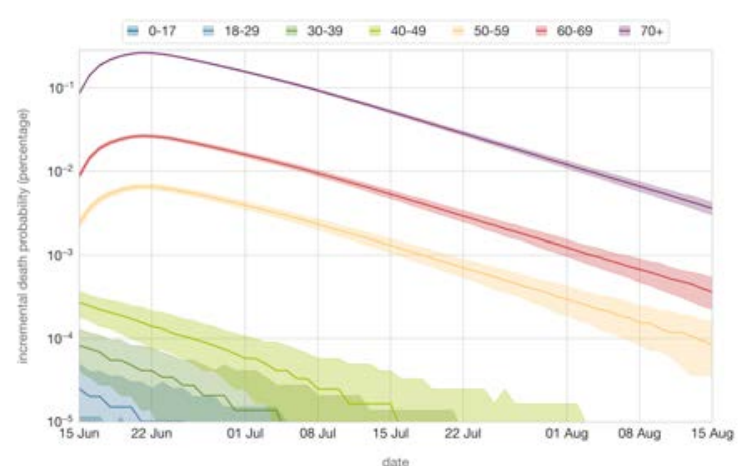
Nadu

\section{Figure A2: Past reproductive rate and consumption and projected death rate.}

Note. (A) Points and line report raw and smoothed seroprevalence-scaled infection rate, respectively. (B)

Household consumption is obtained from CMIE's CPHS through October 2020. Consumption is relative to January 2019 consumption in the location indicated. Mobility is obtained from Google mobility reports and runs from February 2020 to January 2021. Average of relative non-residential mobility is plotted. (C) Assumes no vaccination. 


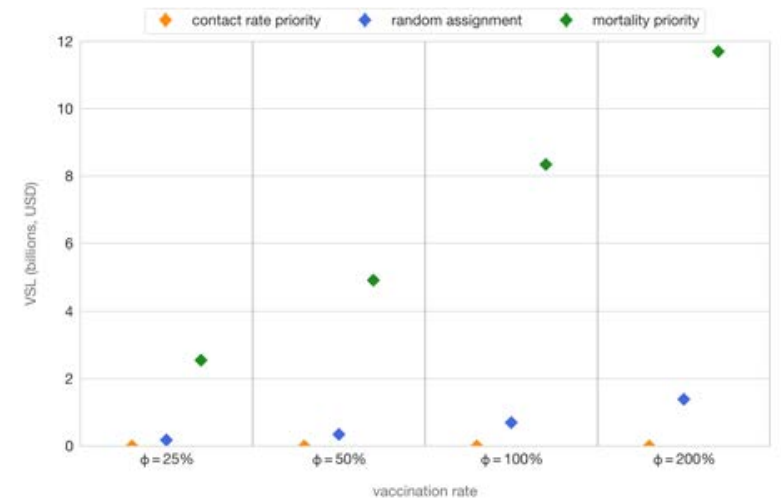

(a) VSL

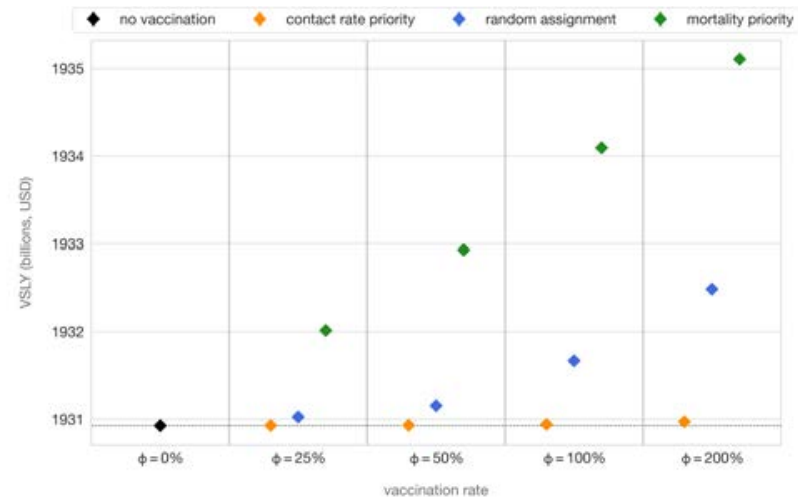

(b) VSLY

\section{Figure A3: Value from different vaccination strategies in India.}

Note. Each whisker plot gives the median and range of outcomes across 1,000 simulations per vaccination policy. Vaccination rates $(\phi)$ are the fraction of a district vaccinated each year. Vaccine efficacy is assumed to be $70 \%$. Policies simulated include no vaccination $(\phi=0)$ and different prioritization schemes and vaccination rates. 